\title{
A general approach for stabilizing nanobodies for intracellular expression
}

2 John Dingus, Jonathan C.Y. Tang*, Constance Cepko

3 Howard Hughes Medical Institute, Blavatnik Institute, Harvard Medical School, United States ${ }^{*}$ currently at Columbia University, United States

\section{Abstract}

Conventional antibodies and their derived fragments are difficult to deploy against intracellular targets in live cells, due to their bulk and structural complexity. Nanobodies provide an alternative modality, with well documented examples of intracellular expression. Despite their promise as intracellular reagents, there has not been a systematic study of nanobody intracellular expression. Here, we examined intracellular expression of 75 nanobodies from the Protein Data Bank. Surprisingly, a majority of nanobodies were unstable in cells, illustrated by aggregation and clearance. Using comparative analysis and framework mutagenesis, we developed a general approach that stabilized a great majority of originally intracellularly unstable nanobodies, without compromising target-binding function. Additionally, we identified distinct sequence features impacting nanobody intracellular stability, and demonstrated mutationally stabilized nanobody expression in vivo, in the murine retina and in E. coli. This work will allow for standardized nanobody engineering for intracellular applications, potentiating a growing field of intracellular interrogation and intervention.

\section{Introduction}

Nanobodies, amino terminal fragments derived from a special class of antibody lacking light chains ${ }^{1}$, are the smallest antibody derivatives that retain full antigen binding function. Composed of a single variable domain of the heavy chain $\left(\mathrm{V}_{H} \mathrm{H}\right)$, nanobodies boast several features that make them attractive tools for a range of applications. As monomers, they are versatile building blocks for protein engineering. Due to their compact binding interfaces, they have become invaluable as protein crystallization chaperones to resolve high resolution crystal structures $^{2,3,4}$. Their modular serum half-life and superior tissue penetration are attractive characteristics for the development of therapeutic biologics ${ }^{5,6}$. Perhaps one feature that has been taken somewhat for granted is superior stability that facilitates nanobody intracellular expression. Full length antibodies, as well as bulkier antibody fragments, are not normally amenable to intracellular expression, partially owing to the reducing environment of the 
protein complexes and signaling pathways. Furthermore, intracellular nanobodies can be directed against novel therapeutic targets previously inaccessible to biologics.

Several groups have detailed intracellular nanobody expression to facilitate live imaging of subcellular factors ${ }^{7,8,9}$. However, few have commented on intracellular expression of nanobodies as a class as it pertains to stability. Those that have broadly detailed nanobody stability have done so in an extracellular context, in buffered solutions that do not resemble the cytoplasmic environment ${ }^{13,14}$. We have been developing tools for nanobody-mediated, fluorescence-based sorting of live, target expressing cells ${ }^{10}$, and genetic manipulation of cells expressing specific targets ${ }^{11,12}$. As we expanded our initial studies, we found that many nanobodies were not natively stable in the intracellular environment. We thus set out to investigate intracellular expression of nanobodies more systematically. To this end, 75 unique nanobody sequences from crystal structures uploaded to the Protein Data Bank (PDB) were cloned into mammalian expression vectors, fused C-terminally to a fluorescent protein (FP). Transfection and live-cell fluorescence imaging of these nanobody-FP fusions in both 293T and HeLa cells revealed that many nanobodies degrade and/or aggregate within the cytoplasm and nucleus, while others appear stable and soluble (Figure 1A). Following these observations, we set out to define intracellular instability based on sequence features, as well as to derive a standardized method for stabilization of previously intracellularly unstable nanobodies through framework mutagenesis. By leveraging positional sequence conservation apparent across intracellularly stable nanobodies, we have determined a method by which the vast majority of previously intracellularly unstable nanobodies can be stabilized for intracellular expression. This mutational stabilization, first observed in cell lines, was further validated for expression in vivo, in E. coli, and in the murine retina. These findings will contribute to the broader adoption of nanobodies as powerful reagents for both research and therapeutic applications.

\section{Results}

\section{Classification of Intracellularly Stable and Unstable Nanobodies}

To investigate nanobody intracellular expression broadly, we first compiled a repertoire of nanobody sequences to profile. The Protein Data Bank was combed for nanobody crystal structures and sequences, resulting in a list of 75 unique sequences, representing nanobodies derived from 3 camelid species and targeting 44 unique protein targets (sequences and targets detailed in Supplemental Table 1). These sequences were cloned into a mammalian expression vector in frame with TagBFP, linked by a 2 amino acid linker. All 75 sequences were expressed via transient transfection in both 293T and HeLa cells in separate wells of a 96 well plate. Intracellular expression patterns were captured by live fluorescence imaging. 33 out of 75 
sequences exhibited strong, diffuse fluorescent signal, expected of well-expressed and intracellularly stable and soluble protein. Interestingly, 42 out of 75 sequences exhibited intracellular phenotypes suggestive of intracellular instability, including low or absent fluorescent signal coincident with varying degrees of aggregation (Figure 1A). These results were confirmed over several replicate experiments.

After binning the nanobodies into "stable" and "unstable" groups, their sequences were analyzed to identify features that distinguished the two groups. Nanobodies are composed of three variable loops (CDR1-3), responsible for the majority of target-specific interaction, and four framework regions (FR1-4), forming the conserved framework structure of the nanobody. First, the average length of variable loop CDR3 was calculated for each group, as CDR3 loop length is highly variable (between 5 and 26 amino acids for nanobodies in the examined repertoire) and CDR3 represents the area of both the greatest sequence-level and structural diversity across nanobodies. While there was little difference in average CDR3 loop length between the groups (16.4 amino acids for the stable group and 16.9 amino acids for the unstable group), one difference related to CDR3 stood out: 17/75 nanobodies contained a CDR3 cysteine that normally forms a disulfide bond tethering CDR3 to either CDR1 (first variable loop) or FR2 (second framework region); all 17 were in the unstable group (Table 1). Because disulfides do not form when nanobodies are expressed in the cytoplasm, this feature may represent a structural liability for intracellular expression.

We next derived the consensus sequence (the sequence constructed from the most common amino acid at each position across nanobody sequences) of the framework region (the region of sequence from which CDR sequences have been removed) for each group. Sequence level deviation between each stability-binned nanobody and the consensus sequence for stable nanobodies is illustrated in Table 1. Stable and unstable consensus sequences for the framework region were identical except at one position, amino acid 52 (IMGT numbering), where the most common amino acid across stable nanobodies was Phe and across unstable nanobodies was Gly. Interestingly, position 52 is one of four hallmark framework positions cited as differentiating $\mathrm{VHs}$ (heavy chain variable regions of conventional antibodies) and $\mathrm{V}_{\mathrm{H}} \mathrm{Hs}^{2}$ (heavy chain variable regions of heavy-chain-only antibodies, analogous to nanobody). These four positions normally contribute to a hydrophobic interaction interface between VH and VL (light chain variable region) in conventional antibodies, and are substituted for amino acids that increase the hydrophilic character of the surface in $\mathrm{V}_{\mathrm{H}} \mathrm{Hs}$. 52Gly has been shown to enhance nanobody solubility, but at the expense of protein yields in E. coli, which may point to decreased stability $^{15,16}$. 
bioRxiv preprint doi: https://doi.org/10.1101/2021.04.06.438746; this version posted April 8, 2021. The copyright holder for this preprint (which was not certified by peer review) is the author/funder, who has granted bioRxiv a license to display the preprint in perpetuity. It is made available under aCC-BY 4.0 International license.

101

102

103

104

105

106

107

108

109

110

111

112

113

114

Table 1: framework sequence variability (compared to consensus sequence) across stable and unstable nanobodies $\square$ extra disulfide bond

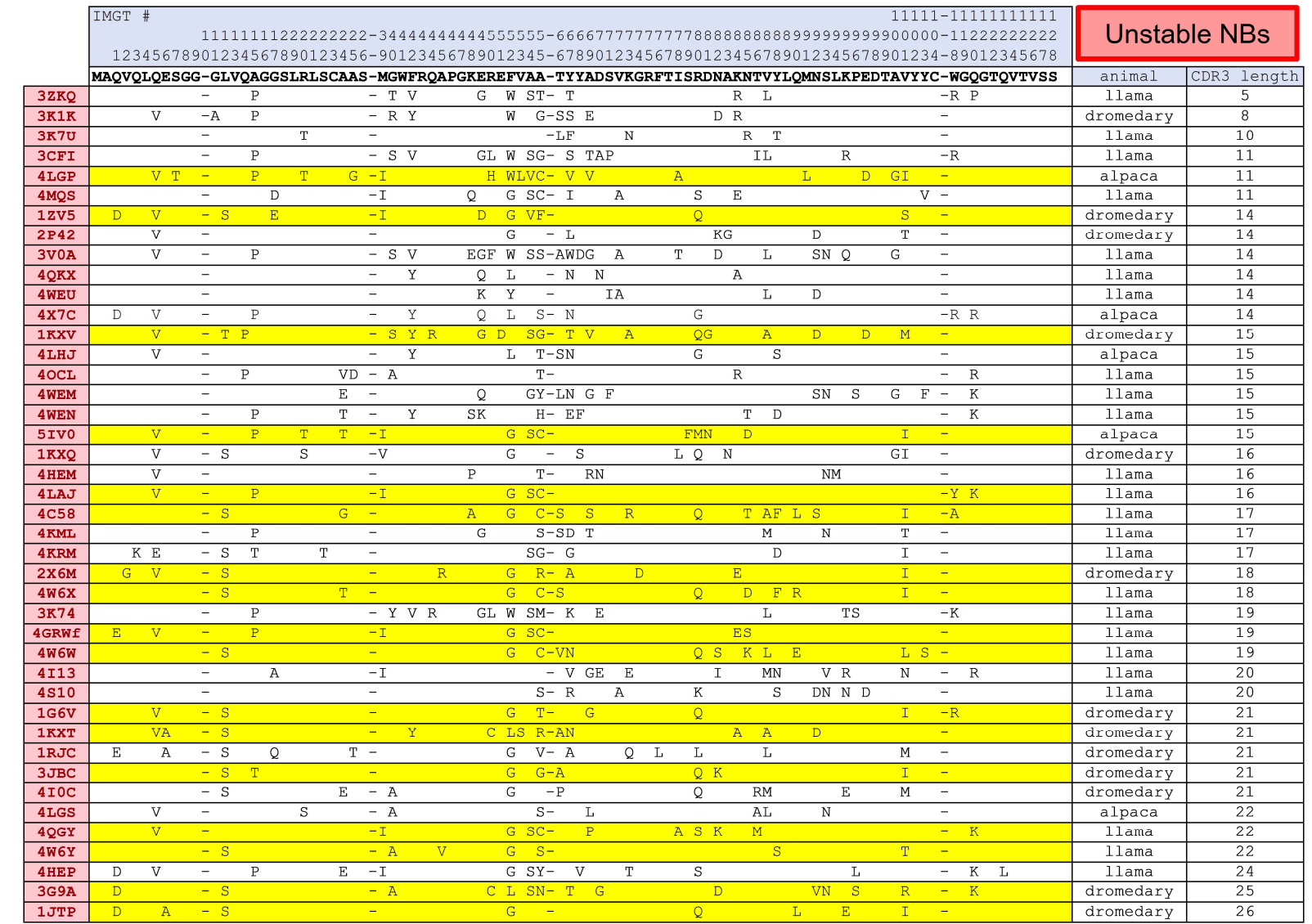

$11111111222222222-34444444444555555-6666777777777788888888889999999999900000-11222222222$

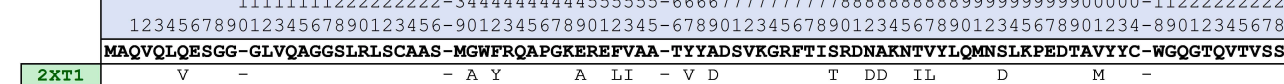

Stable NBs

116

117

118

119

120

121

122

123

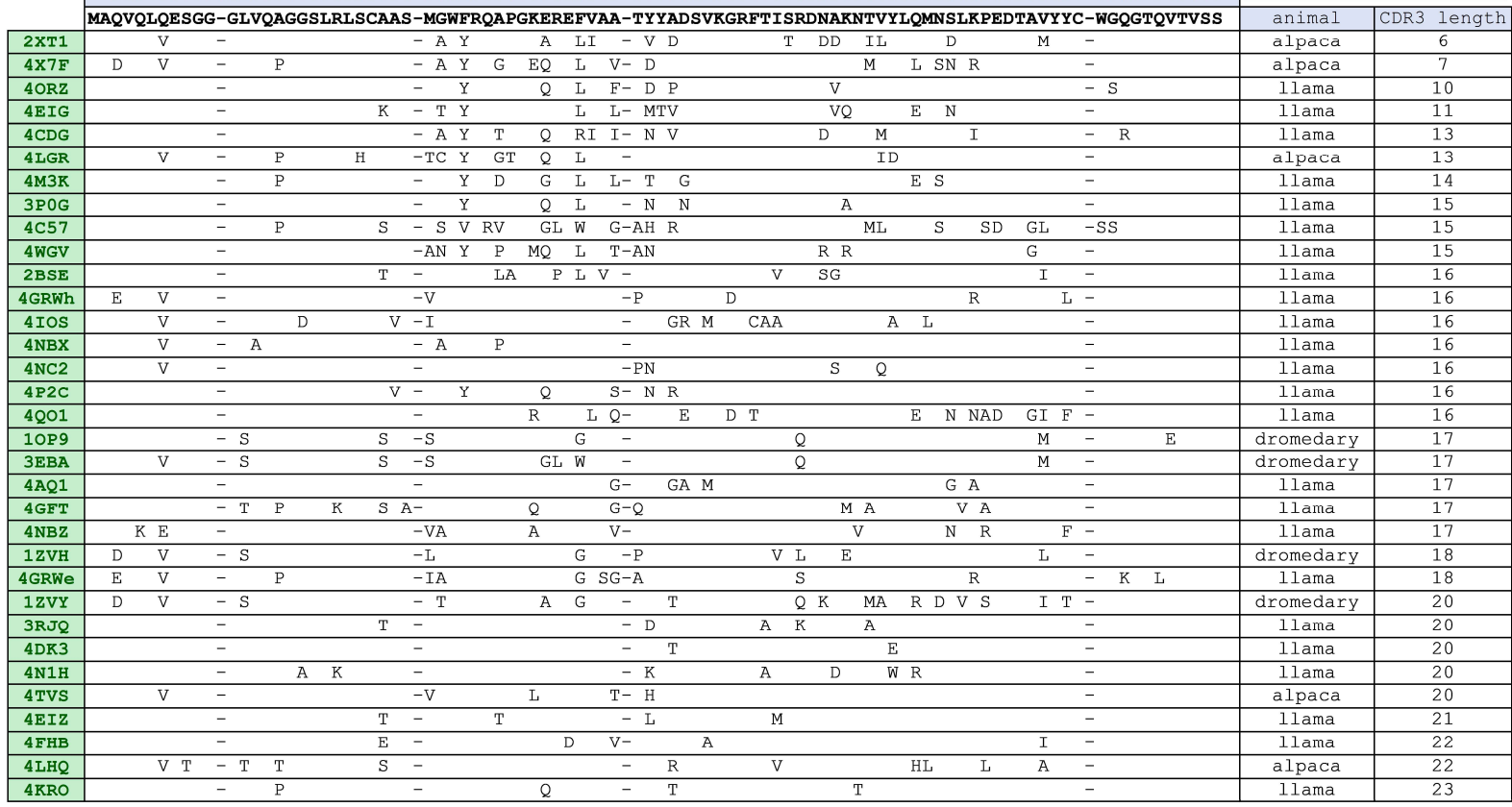

*all nanobodies contain a conserved set of cysteines that normally forms a disulfide bond through the hydrophobic core of the

126 nanobody (23Cys and 104Cys). Highlighted in yellow, "extra disulfide bond" refers to nanobodies with an additional pair of cysteines, one of which is always located in CDR3, that normally forms a disulfide bond that impacts CDR3 conformation. 
130
B

\begin{tabular}{|c|c|c|c|c|c|}
\hline \multicolumn{3}{|c|}{ Stable Enriched $(p<.05)$} & \multicolumn{3}{|c|}{ Unstable Enriched $(p<.05)$} \\
\hline Residue & Events & $\underline{P \text {-value }}$ & Residue & Events & $\underline{P \text {-value }}$ \\
\hline $12 \mathrm{~L}$ & 52 & 0.036 & $12 S$ & 19 & 0.014 \\
\hline \multirow[t]{3}{*}{$24 S$} & 5 & 0.014 & & & \\
\hline & & & 391 & 11 & 0.048 \\
\hline & & & 45A & 63 & 0.007 \\
\hline $49 Q$ & 11 & 0.033 & & & \\
\hline $52 \mathrm{~F}$ & 27 & 0.016 & $52 G$ & 23 & 0.002 \\
\hline 54A & 56 & 0.001 & $54 S$ & 14 & 0.002 \\
\hline \multirow[t]{2}{*}{ 55A } & 25 & 0.001 & $55 \mathrm{C}$ & 9 & 0.004 \\
\hline & & & $66 S$ & 5 & 0.049 \\
\hline 73K & 69 & 0.026 & & & \\
\hline $80 R$ & 50 & 0.015 & & & \\
\hline $120 Q$ & 62 & 0.030 & & & \\
\hline
\end{tabular}

IMGT

11111-11111111111 $11111111222222222-34444444444555555-666677777777778888888888999999999900000-11222222222$ $12345678901234567890123456-90123456789012345-678901234567890123456789012345678901234-89012345678$ MAQVQLQESGG-GLVQAGGSLRLSCAAS-MGKF RQAPGKEREFVAA-TYYADSVKGRFTISRDNAKNTVYLQMNSLKPEDTAVYYC-WGQGTQVTVSS

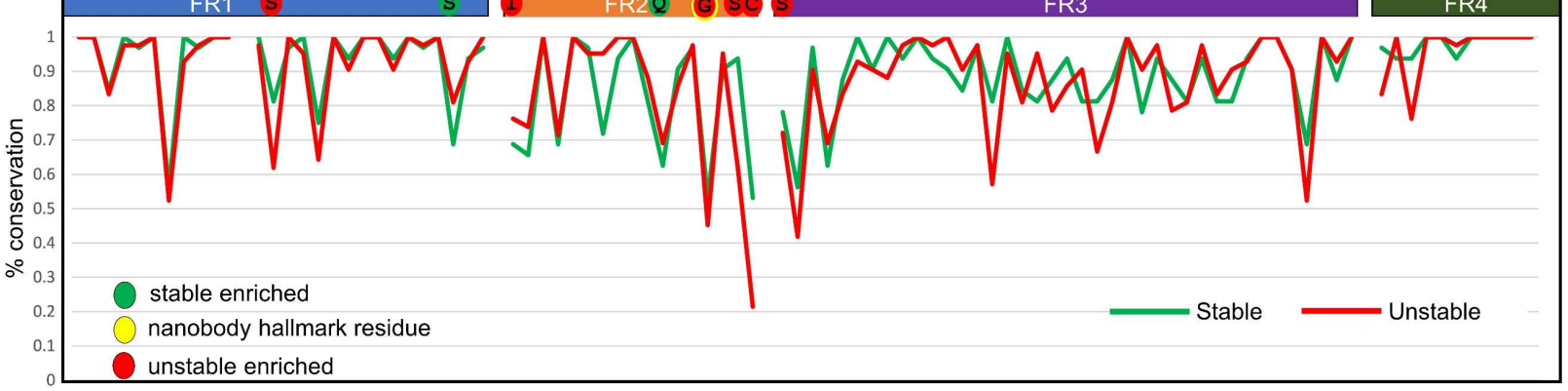

Figure 1| Sequence differences between intracellularly stable and unstable nanobodies

(A) Representative images of intracellularly stable and unstable nanobody-TagBFP fusions in transiently transfected 293T cells. Red signal is from co-transfected CAG-dsRed plasmid. (B) Significantly enriched positional residues between stable and unstable nanobodies (Fisher's exact test). Events denote the total number of instances of each positional residue across all nanobodies (75 total). Grey cells denote positional insignificance. (C) Positional amino acid conservation across framework sequences of stable and unstable nanobodies. Scale bar is $20 \mu \mathrm{m}$.

Next, we examined total positional enrichment for each amino acid across each position for both stable and unstable nanobody frameworks. Applying a Fisher's exact test, 12 framework positions with residues that were significantly enriched in either group were identified, with 4 of those positions having a specifically enriched residue in both groups (Figure 1B). Of note, 52Gly was strongly enriched in the unstable group (19/23 occurrences in unstable nanobodies), as well as 55Cys, a cysteine that normally forms a disulfide bond with a CDR3 cysteine in a subset of nanobodies, as noted above (9/9 occurrences in unstable nanobodies). Additionally, 54Ser emerged as a strongly enriched unstable residue likely to drive instability (13/14 occurrences in unstable nanobodies). Position 54 points its functional group into the 
160

161

162

163

164

165

166

167

168

169

170

171

172

173

174

175

176

177

178

179

180

181

182

183

184

185

186

187

188

189

190

191 hydrophobic core of the nanobody, and is usually occupied by a small hydrophobic amino acid (93\% of stable nanobodies examined contain 54Ala). A hydrophilic substitution at position 54 is likely to contribute to destabilization of the hydrophobic core.

Each unstable nanobody had at least 1 of 7 identified positional residues significantly enriched in the unstable group. However, the most common positional residue enriched in the unstable group, 45Ala, is the most common residue at that position for both groups, and is therefore unlikely to be a strong driver of instability (40/42 unstable nanobodies and 24/33 stable nanobodies contain 45Ala). 10/42 unstable nanobodies had 45Ala as their only enriched residue. Since this preliminary sequence analysis was unlikely to comprehensively explain the differences between stability groups, we decided to apply a broad mutagenesis strategy to unstable nanobodies to try to achieve stability.

\section{Stabilizing Mutagenesis Based on Positional Conservation}

We set out to define a general mutagenesis approach to stabilize most, if not all, nanobodies for intracellular expression, taking advantage of sequence features observable across expression-profiled nanobodies. At the outset, our strategy was based upon the assumption that sets of important stabilizing residues are likely to be highly conserved across intracellularly stable nanobodies. While the framework consensus sequences for stable and unstable nanobodies are nearly identical, positional conservation varies between the two groups (Figure 1C). Our approach for conservation-based mutagenesis is schematized in Figure 2A. A threshold of $\geq 80 \%$ positional conservation was applied to generate a partial consensus sequence of the most highly conserved positional residues across stable nanobodies. This partial consensus framework was applied as a filter to identify non-conforming positional residues in each unstable nanobody for mutagenesis. At each position that a given unstable nanobody disagreed with the filter, that residue was changed to agree with the filter. An exception was made to maintain any cysteine that normally participates in a disulfide bond, as these cysteines likely impact positioning of CDR3. Applying these changes to all 42 intracellularly unstable nanobodies yielded mutants with a range of between 2 and 12 changes per nanobody, with an average mutational load of 5.7 changes per nanobody. Mutation numbers for each nanobody are reported in Tables $2 \mathrm{~B}$ and $\mathrm{C}$. 
bioRxiv preprint doi: https://doi.org/10.1101/2021.04.06.438746; this version posted April 8, 2021. The copyright holder for this preprint (which was not certified by peer review) is the author/funder, who has granted bioRxiv a license to display the preprint in perpetuity. It is made available under aCC-BY 4.0 International license.

A
Stable Nanobodies

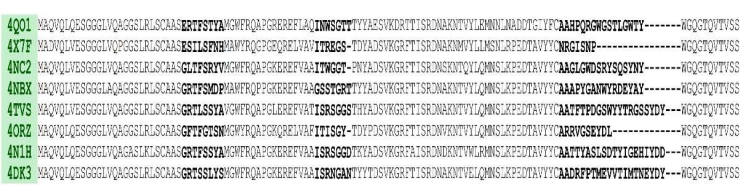

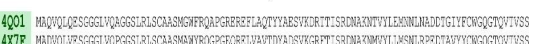

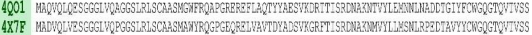

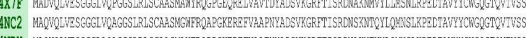

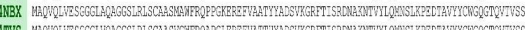

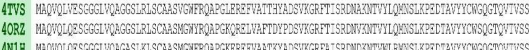

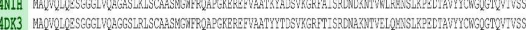

\section{(20)}
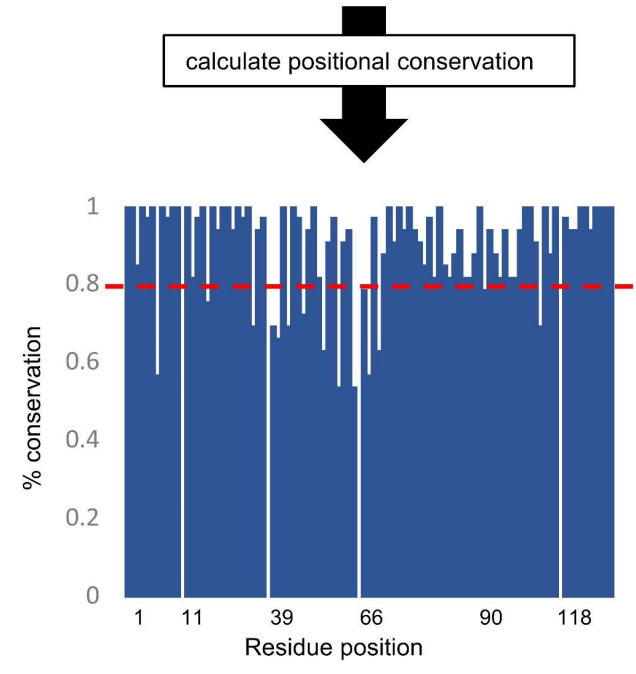

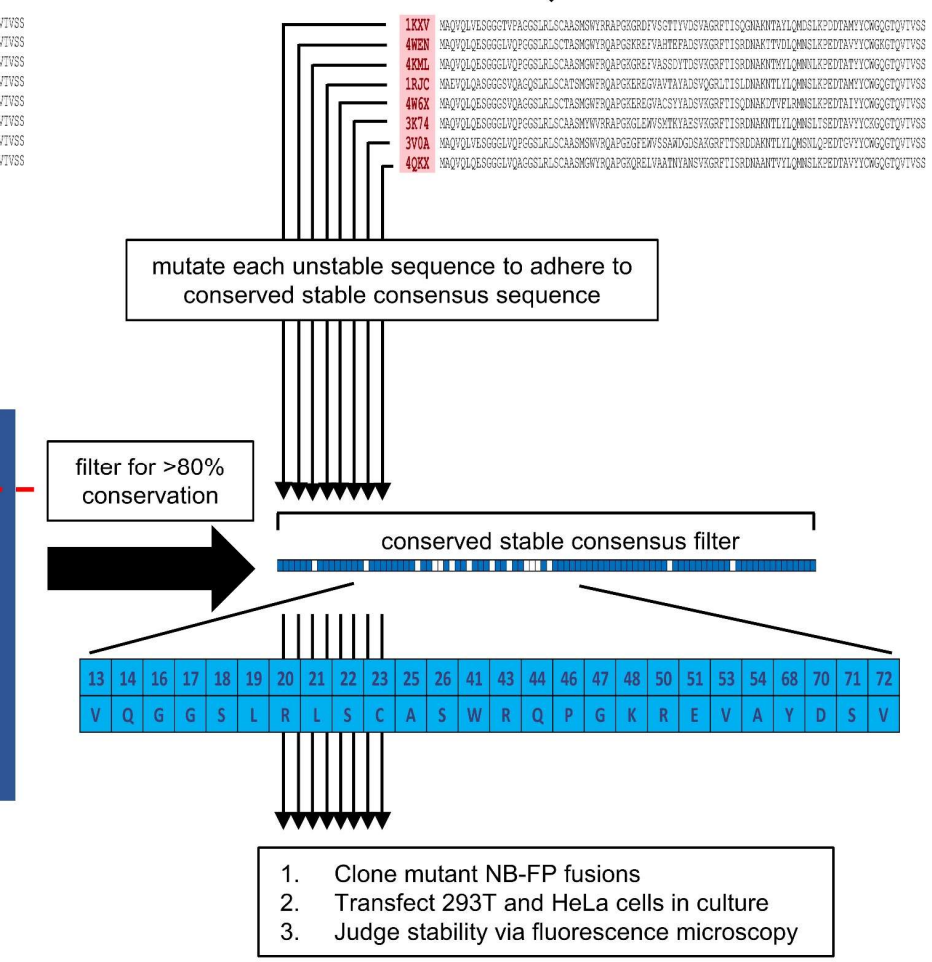

Unstable Nanobodies

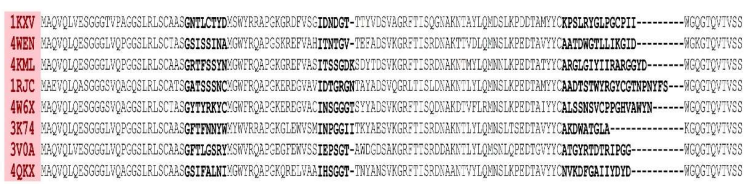

\section{8}

B

221

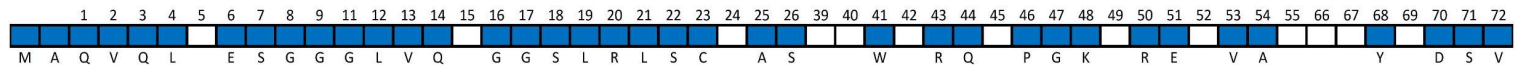

(A) Nanobody-TagBFP fusions were classified as "stable" or "unstable" based on intracellular expression via transient transfection in 293T cells. Amino acid sequences were binned according to stability group, and variable domain sequences (CDRs) were excluded from downstream consideration. Positional sequence conservation was calculated across stable nanobody sequences, and positional amino acids of high conservation $(>80 \%)$ were compiled to form a partial consensus filter. Each individual unstable nanobody framework was then compared to this filter, and any positional amino acid disagreement was resolved to adhere to the filter. Mutated nanobodies were then cloned, transfected into 293T and HeLa cells, and judged for stability via fluorescence microscopy. (B) All positional amino acids captured in the partial-consensus framework (blue cells). White cells denote framework positions excluded from the partial consensus framework. 
bioRxiv preprint doi: https://doi.org/10.1101/2021.04.06.438746; this version posted April 8, 2021. The copyright holder for this preprint (which was not certified by peer review) is the author/funder, who has granted bioRxiv a license to display the preprint in perpetuity. It is made available under aCC-BY 4.0 International license.

Table 2A: pre-mutagenesis framework sequence variability across unstable nanobodies

stable

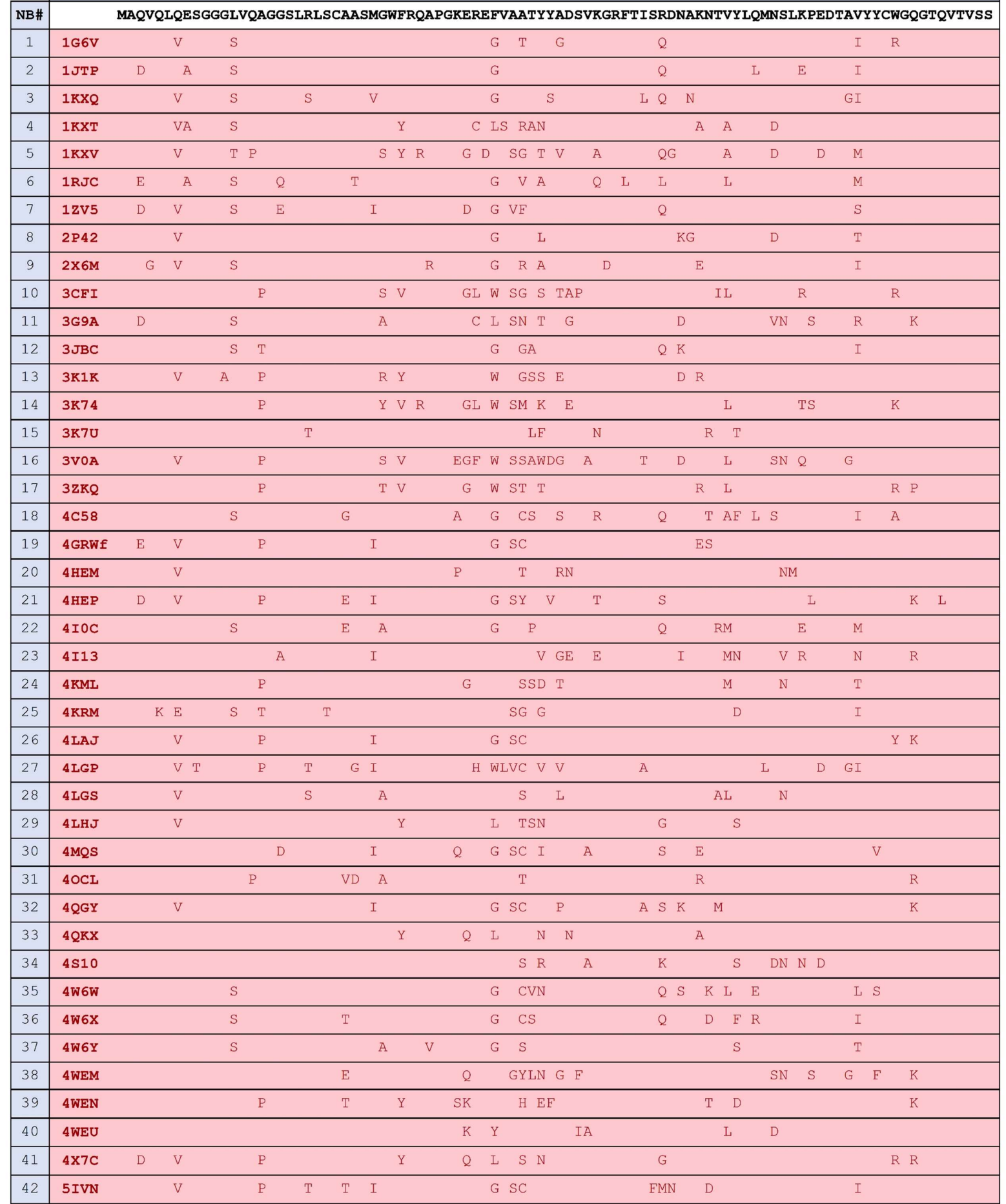


bioRxiv preprint doi: https://doi.org/10.1101/2021.04.06.438746; this version posted April 8, 2021. The copyright holder for this preprint (which was not certified by peer review) is the author/funder, who has granted bioRxiv a license to display the preprint in perpetuity. It is made available under aCC-BY 4.0 International license.

Table 2B: sequence and stability variability across nanobodies following stage 1 mutagenesis

12345678912345678901234569012345678901234567890123456789012345678901234567890123489012345678

MUT\# MAQVQLQESGGGLVQAGGSLRLSCAASMGWFRQAPGKEREFVAATYYADSVKGRFTISRDNAKNTVYLQMNSLKPEDTAVYYCWGQGTQVTVSS

\begin{tabular}{|c|c|c|c|c|c|c|}
\hline 7 & $1 \mathrm{KX} Q$ & V & & V & (G) & I \\
\hline 6 & $1 \mathrm{KXT}$ & V & & Y & C L RAN & \\
\hline 11 & $1 \mathrm{KXV}$ & V & & S Y & G $\quad G \quad T \quad V$ & M \\
\hline 4 & $2 \times 6 M$ & V & & & $\mathrm{R} A$ & I \\
\hline 8 & 3CFI & & P & $\mathrm{S}$ & 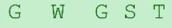 & \\
\hline
\end{tabular}

\begin{tabular}{|c|c}
\hline 9 & $3 \mathrm{G} 9$ \\
\hline 4 & $3 \mathrm{~K} 1$ \\
\hline 9 & $3 \mathrm{~K} 7$ \\
\hline
\end{tabular}

G9A A $\quad$ A $\quad$ C L $\quad$ N T

\begin{tabular}{|c|lllllllll}
\hline 4 & 3R1K & V & P & R & Y & & W & G \\
\hline 9 & 3R74 & & P & Y & G & W & M \\
\hline 13 & 3V0A & V & P & S & G & W & S \\
\hline 6 & 3ZRQ & & P & & T & G & W & T \\
\hline 4 & 4 GRFf & V & P & & I & & G & C \\
\hline 8 & 4 HEP & V & P & E & I & & G & Y \\
\hline 9 & 4 I13 & & & & & I & & &
\end{tabular}


273

274

275

276

277

278

279

280

281

282

283

284

285

286

287

288

289

290

291

292

293

294

295

296

297

298

299

300

301

Table 2C: sequence and stability variability across nanobodies following stage 2 mutagenesis $(+\mathrm{G} 52 \mathrm{~F}+\mathrm{X} 90 \mathrm{Q})$

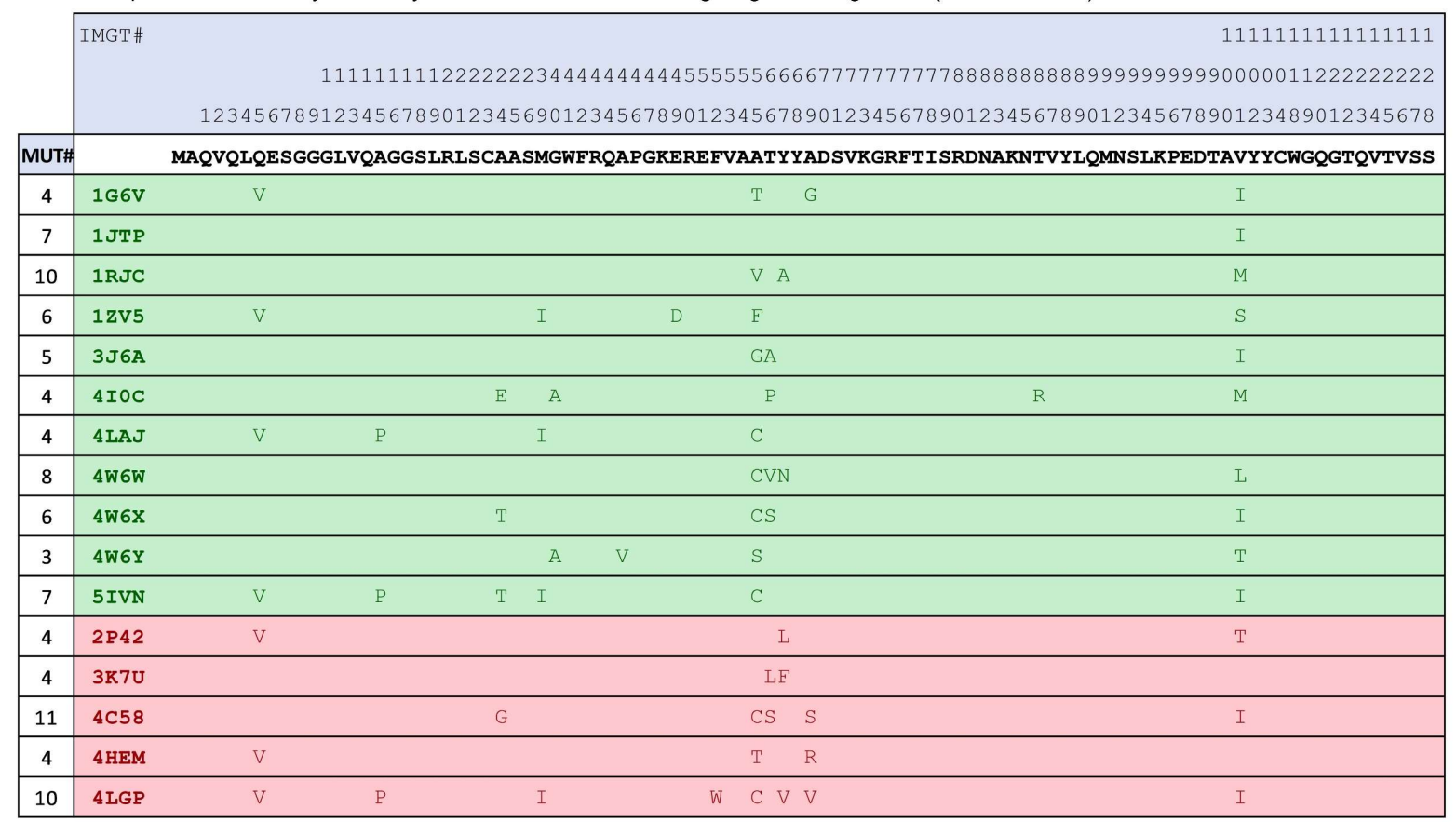

We cloned the derived mutant nanobodies and expressed them as FP fusions in 293T and HeLa cells as we had expressed their wild-type parents, previously. Sequence variability before and after mutagenesis for unstable nanobodies, as well as stability status following mutagenesis, is illustrated in Table 2. Fluorescent imaging revealed that 26/42 nanobodies had been effectively stabilized via targeted mutagenesis, as illustrated by strong fluorescent signal and a total absence of punctate aggregates (Figure 3A,C). Another 6/42 nanobodies exhibited improvement compared to their wild-type parent with respect to aggregation levels and soluble protein fraction. 10/42 nanobodies exhibited no improvement from mutagenesis.

Aligning the 42 mutant sequences, grouping sequences that were effectively, partially, or not stabilized via mutagenesis, a striking correlation emerged: 13/16 nanobodies that were not effectively stabilized via mutagenesis had 52 Gly (Table $2 \mathrm{~B}$ ). Because position 52 is a relatively variable framework position, it was not included in the conservation filter for first pass mutagenesis. Additionally, non-consensus residues at positions 90 and 101 were enriched in nonstabilized nanobodies. For nanobodies not effectively stabilized by stage 1 mutagenesis, up to two additional changes were made wherever possible, Gly52Phe and X90GIn, to try to achieve stability. Because deviation at position 101 was almost perfectly correlated with the presence of Gly52, a likely driver of instability, this position was not changed (Table 2). As a result of this additional round of mutagenesis, $11 / 13$ additional nanobodies were effectively stabilized. In total, 
302

303

304

305

306

307

308

309

310

311

312

313

314

315

316

317

318

319

320

321

322

323

324

325

326

327

328

329

330

331

332

$37 / 42$ previously intracellularly unstable nanobodies were rendered stable and soluble (Figure $3 C)$.

$\underline{\text { Identification of Strong Drivers of Intracellular Stability }}$

Encouraged by the success of the stabilizing mutagenesis approach, we set out to identify specific drivers of stability from amongst the partial consensus framework. To do this, we sought to eliminate "passenger" mutations for individual stabilized nanobodies and to identify the minimal, necessary mutational subsets required for intracellular stabilization. Five mutationally stabilized nanobodies with high mutational load and the high-confidence stability driver Ser54Ala were chosen for further examination (3K74, 3V0A, 3G9A, 4MQS, and 1KXV). $1 \mathrm{KXT}$, a mutationally stabilized nanobody receiving the unique mutation Ser53Val, was also examined as both positions 53 and 54 point inward to the hydrophobic core of the nanobody. Subsets of the original mutations imposed to achieve stability were chosen, and new mutant variants were generated for intracellular stability testing. Mutation subsets were chosen based on crystal structure data and our own stabilization statistics. Several variants are depicted in Figure 3D and E. After testing several mutation variants for each nanobody, Ser54Ala was found to be sufficient to stabilize $3 K 74$ on its own (originally stabilized with 8 mutations). Ser53Val similarly stabilized 1KXT by itself. 3V0A and 4MQS required only Ser54Ala and one additional mutation each to achieve stability (originally stabilized with 11 and 7 mutations, respectively). The minimal necessary sets of mutations needed to stabilize 3G9A and $1 \mathrm{KXV}$ were not identified, suggesting that several of their original mutations, in addition to Ser54Ala, were necessary for stabilization.

68Tyr and 80Arg were identified in nanobodies 3V0A and 4MQS (respectively) as important for their stabilization. These residues were subsequently investigated for their role in the stabilization of 3 additional nanobodies not originally containing the strong instability driver 54Ser: 4WEN, 4LHJ, and 1KXQ. 4LHJ was originally stabilized from only 2 mutations (GIn80Arg and Ser88Tyr). However, removal of Ser88Tyr did not destabilize 4LHJ, illustrating the stabilizing influence of 80Arg alone (data not shown). Surprisingly, 4WEN was effectively stabilized by 68Tyr alone, when it had originally received 5 mutations (Figure $3 \mathrm{D}$ and $\mathrm{E}$ ). $1 \mathrm{KXQ}$, which originally received both Ser68Tyr and GIn80Arg mutations in addition to 5 others, was effectively stabilized by 68Tyr alone, again illustrating the importance of 68Tyr for stability and suggesting only circumstantial importance of 80Arg (data not shown). 


\section{Mutationally Stabilized Nanobodies Retain Target Binding Function in Cells}

It is crucial for stabilizing mutagenesis to not only facilitate intracellular expression, but to maintain the nanobody's ability to bind its target. Conventional antibodies engage their targets in a largely stereotyped fashion, relying heavily on variable loops for target interaction, with minimal direct framework contribution. In contrast, nanobodies exhibit a greater paratope diversity, and contribution of framework residues to binding is more common ${ }^{17}$. We compiled crystal structure data, curated by EMBL-EBI and made available through PDBe PISA, describing the interaction interfaces between nanobodies and their targets. Examination of these interfaces revealed that the great majority of interacting residues across nanobody framework regions are located in the most highly variable framework positions, positions omitted from our conservation mutagenesis approach (Figure 4A). While the possibility that mutation of non-interacting residues will generate conformational changes that impact target binding cannot be ruled out, this finding suggests that our approach likely minimizes impact on target binding, compared to strategies that rely on grafting variable loops onto established nanobody frameworks ${ }^{18}$.

We chose 6 mutationally stabilized nanobodies whose targets are easily expressed intracellularly to test for intracellular target binding (3 targets, 2 nanobodies per target). Each nanobody was C-terminally linked with an FP, and each target was engineered to contain an $\mathrm{N}$ terminal nuclear localization sequence (NLS). Wild-type and mutant nanobodies were transfected with either empty vector or NLS-target into 293T and HeLa cells. Target binding was assessed based on nuclear localization of nanobody-FP signal in the presence of NLS-target, observed via fluorescence imaging.

All 6 stabilized mutants were able to bind target (Figure 4B, Supplemental Figure 1). Of note was that 3G9A, which has a large CDR3 loop that is normally anchored by an extra disulfide bond to FR2, was able to bind target despite assumed loss of the disulfide bond. Interestingly, 2 nanobodies, 3K74 and 4I13, which are unstable in their wild-type forms in the absence of target, exhibited robust target binding in both their wild-type and mutationally stabilized forms. This suggested that the presence of their target, E. coli dihydrofolate reductase (DHFR), had a stabilizing effect (Figure 4B, Supplemental Figure 1). Our lab has previously described nanobody conditional stability in an engineered context ${ }^{10}$, and it is expected for a subset of nanobodies to exhibit this target-dependent stability naturally. 
A

370

371

372

373

374

375

376

377

378

379

380

381

382
B

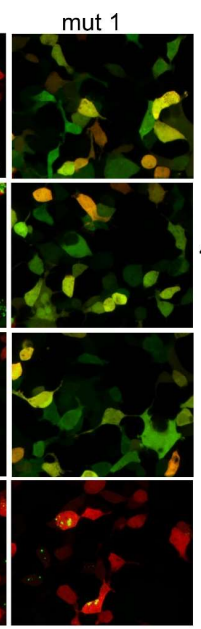

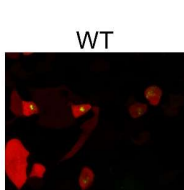

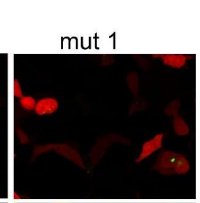

+G52F $+\mathrm{X} 90 \mathrm{Q}$

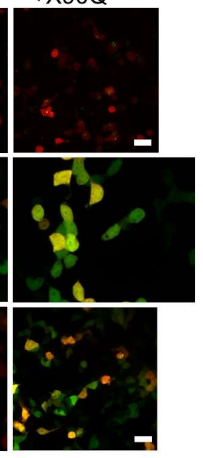

E

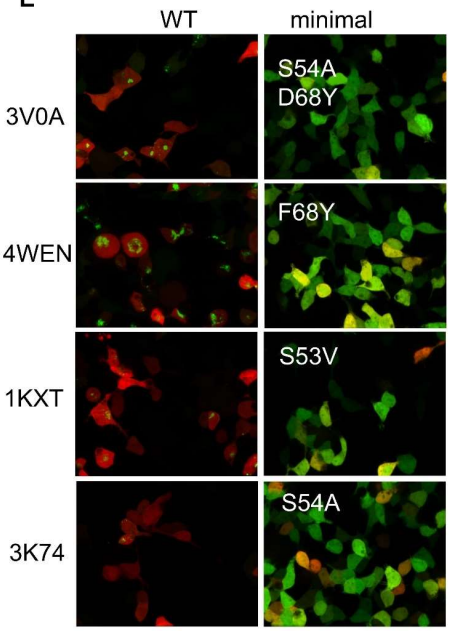

D

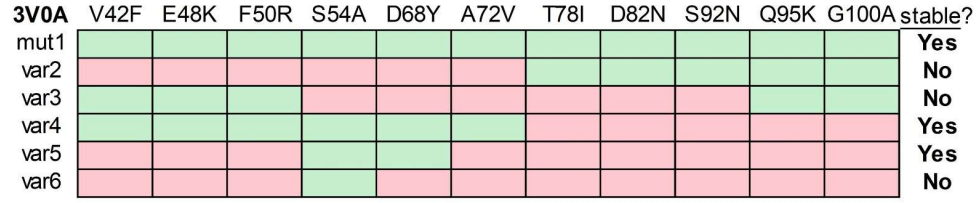

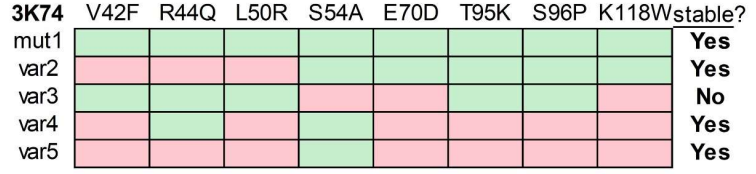

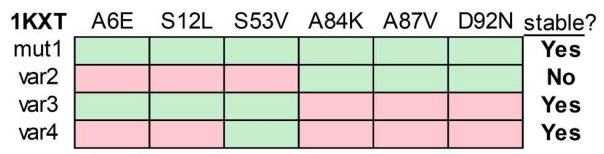

4WEN S48K F68Y T85N D88Y K120Qstable?

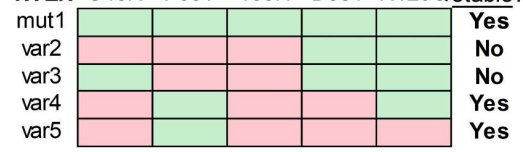

keep mutation

omit mutation

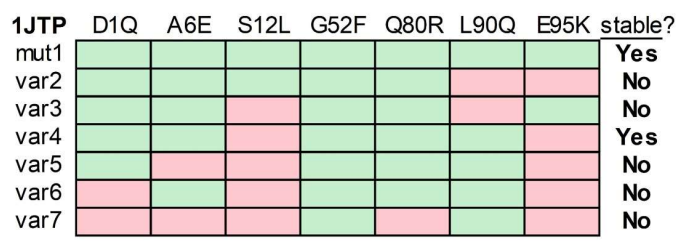

Figure 3| Mutationally stabilized nanobodies and specific stability drivers

(A) Examples of nanobody-TagBFP expression in 293T cells following transient transfection before (WT) and after (mut1) stage 1, conservation-based mutagenesis. Red signal is from co-transfected CAG-dsRed plasmid. (B) Examples of nanobody-TagBFP expression in 293T cells following transient transfection for nanobodies with Gly52. Wild-type, firstpass conservation-based mutants (mut1), and mutants with up to 2 additional mutations, Gly52Phe and X90GIn, are depicted. (C) Numbers of stable vs unstable nanobodies (75 total) following first-pass conservation-based mutagenesis (stage 1 mut) and final mutagenesis $(+G 52 F+X 90 Q$ ). (D) Example mutant variants tested for specific mutationallystabilized nanobodies in order to identify necessary stability mutations. (E) Examples of nanobody-TagBFP expression in 293T cells following transient transfection for nanobodies effectively stabilized by 1-2 mutations. All scale bars are $20 \mu \mathrm{m}$. 
398

399

400

401

402

403

404

405

406

407

408

409

410

411

412

413

414

415

416

417

418

419

420

421

422

423

424

425

426

427

428

429

We next wanted to evaluate the necessity of using a partial consensus strategy for stabilization (Figure 2B), in which only highly conserved framework residues are fixed, as opposed to a full consensus strategy in which all framework residues are fixed (Figure 5A). The above 6 nanobodies were therefore tested for stability and target-binding after grafting their variable loops onto full consensus frameworks (50Cys was maintained for 3G9A). 6/6 nanobodies with full consensus frameworks exhibited stable expression in cells (Figure 5B). However, 2/6 nanobodies that achieved target binding in the context of our partial consensus framework (Figure 4B) lost binding in the context of the full consensus framework (Figure 5B). These nanobodies, $3 \mathrm{~K} 1 \mathrm{~K}$ and $3 \mathrm{~K} 74$, normally engage their targets along a concave framework interface (Figure 5C). Specifically, residues across FR2 form direct contacts with target, representing a common mode of binding (Figure 5C and D).

We next turned our attention to a nanobody with an atypical mode of target binding. Nanobody 5IVN (also known as nanobody BC2) targets a short N-terminal stretch of $\beta$-catenin, representing a rare epitope: a short, linear peptide amenable for use as an affinity tag. Corroborating previous findings, we found 5IVN to be unstable when expressed in cells ${ }^{7}$. Unfortunately, while our mutagenesis approach stabilized 5IVN for cellular expression, it did not facilitate intracellular binding of its epitope as assessed by lack of co-localization with its target following cellular co-transfection of 5IVO-TagBFP and NLS-mCherry-epitope (data not shown). Binding was not achieved after limiting stabilizing mutagenesis to the single, necessary mutation to achieve intracellular stability, Ser54Ala. We suspect this lack of binding relates to loss of a crucial extra disulfide bond that normally orients its highly conformationally-precise paratope, but that does not form intracellularly. This disulfide bond has previously been shown to be required for target binding ${ }^{19}$.

Taken together, these target binding experiments suggest that nanobodies stabilized by the conservation-based mutagenesis approach developed here are likely to retain target binding function in cells, although certain conformational paratopes may not be amenable for intracellular recapitulation. 
bioRxiv preprint doi: https://doi.org/10.1101/2021.04.06.438746; this version posted April 8, 2021. The copyright holder for this preprint (which was not certified by peer review) is the author/funder, who has granted bioRxiv a license to display the preprint in perpetuity. It is made available under aCC-BY 4.0 International license.

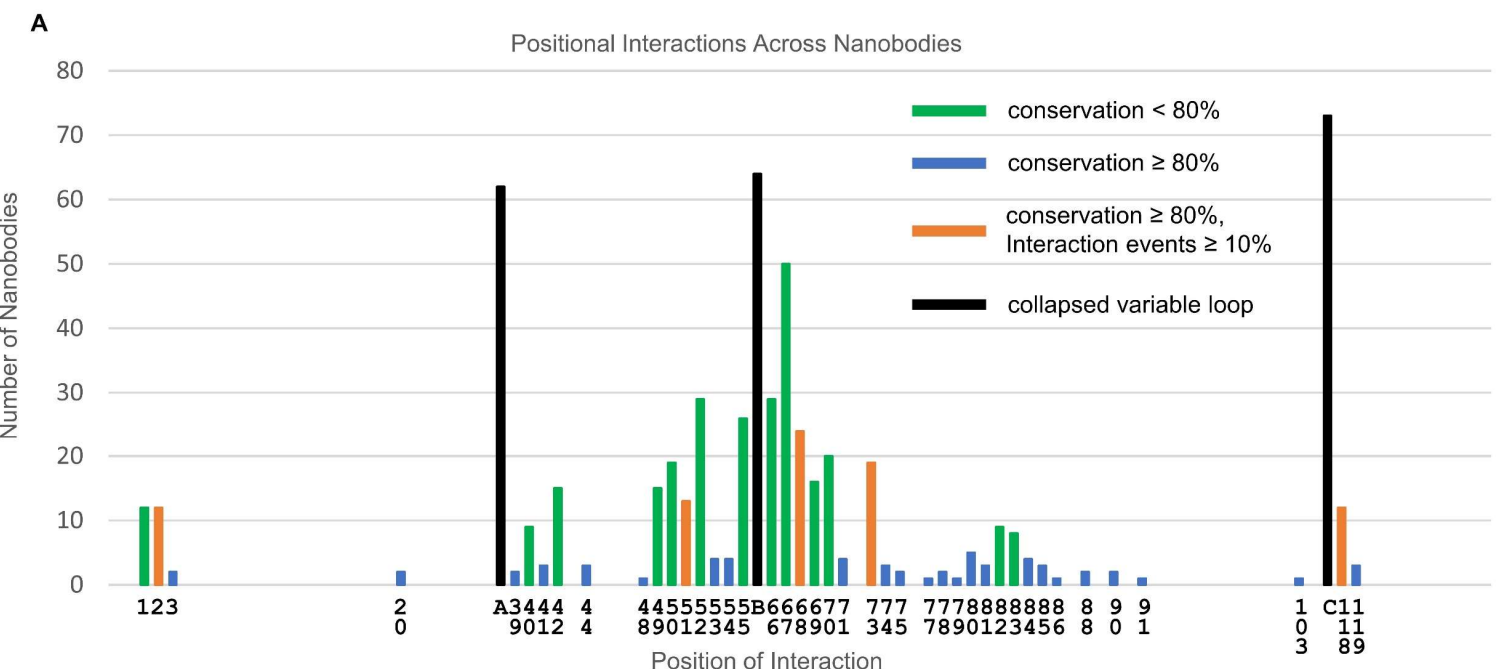

B
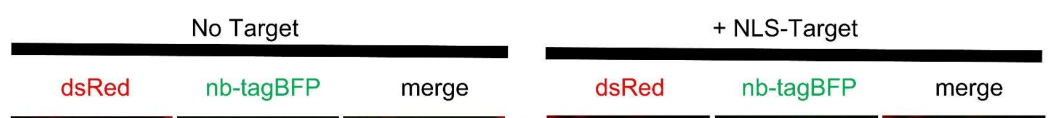

3G9A mut
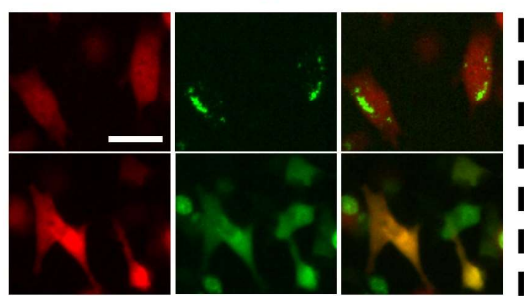

4WEM WT

3K74 WT
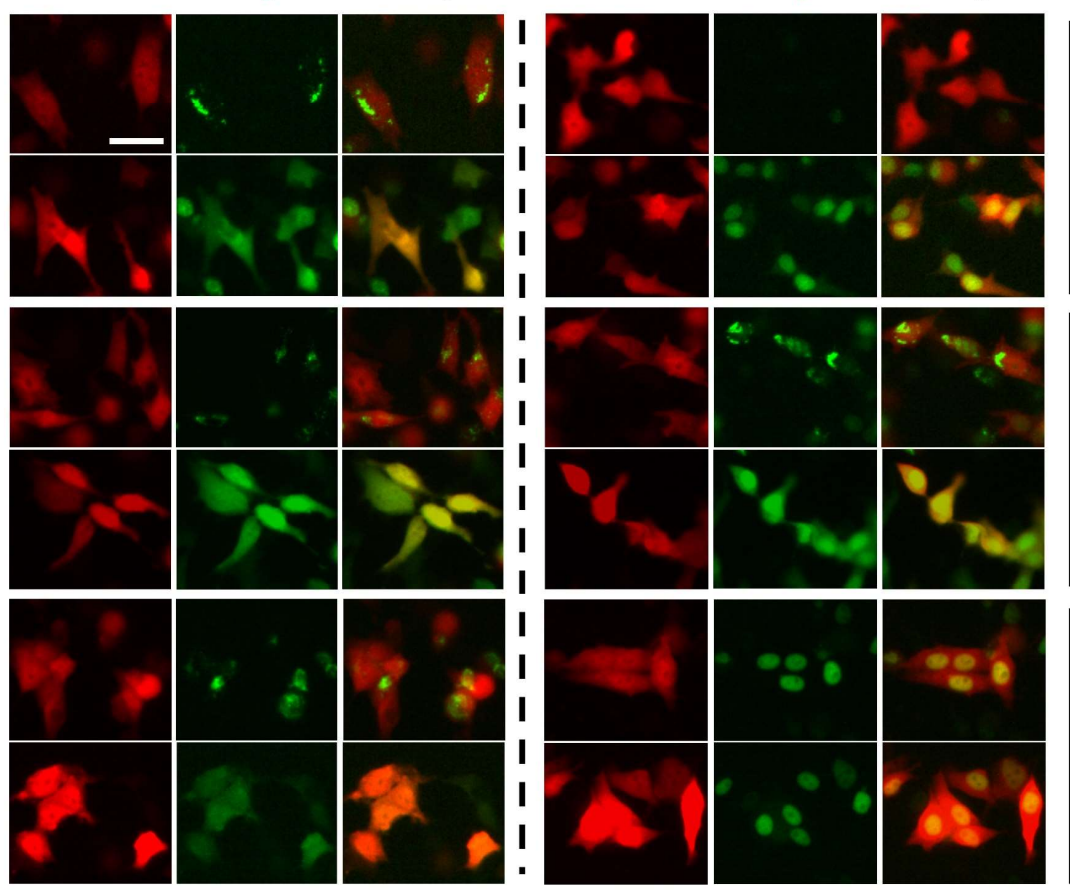

Anti-GFP
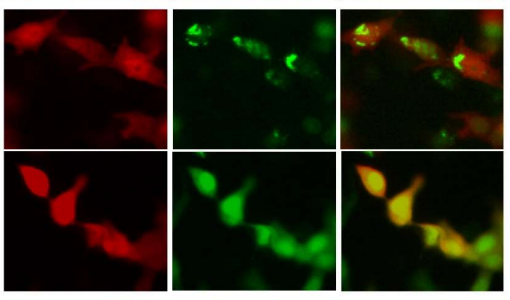

Anti-faeg
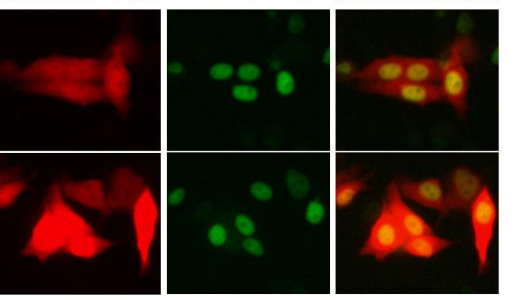

Anti-DHFR

(E. coli)

\section{Figure 4| Target binding of parent and mutationally stabilized nanobodies}

(A) Total number of target-interfacing positions across 75 nanobodies. Positions of interaction were determined based on buried surface area for crystal structure interfaces made available through PDBE-PISA. Black bars labelled "A, B, and C" denote positions across CDRs 1,2 and 3 , respectively. Colored bars describe sequence-level conservation across targetinterfacing framework positions. (B) Representative images of nanobody-TagBFP expression in HeLa cells in the presence and absence of nuclearly localized-target. Wild-type and mutant nanobodies are depicted. Red signal is from co-transfected CAG-dsRed plasmid. Transfected DNA amount was normalized by addition of empty vector plasmid to transfection mix for the "no target" condition. Scale bar is $25 \mu \mathrm{m}$. 
bioRxiv preprint doi: https://doi.org/10.1101/2021.04.06.438746; this version posted April 8, 2021. The copyright holder for this preprint (which was not certified by peer review) is the author/funder, who has granted bioRxiv a license to display the preprint in perpetuity. It is made available under aCC-BY 4.0 International license.

A

B

463

464

465

466

C

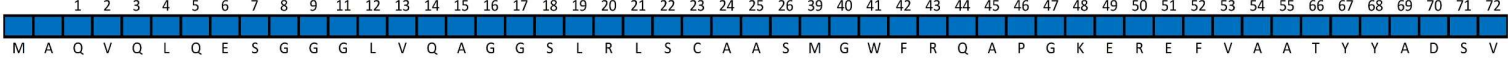

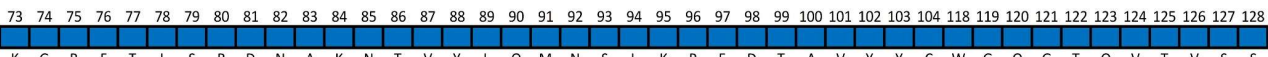

No Target

+ NLS-Target

dsRed nb-tagBFP merge

dsRed nb-tagBFP merge

3G9A con
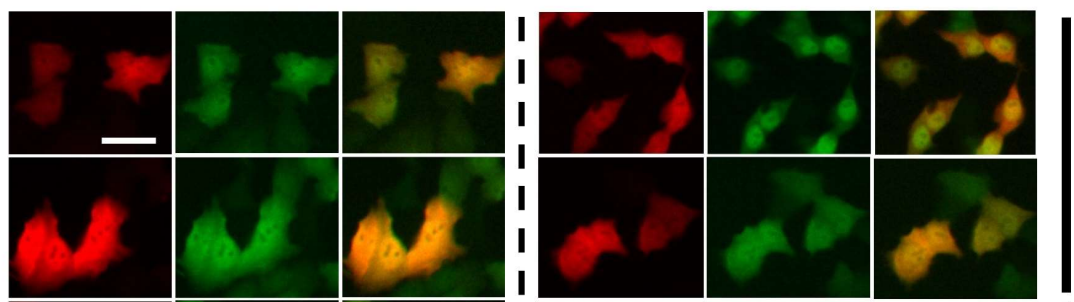

Anti-GFP

4WEM con
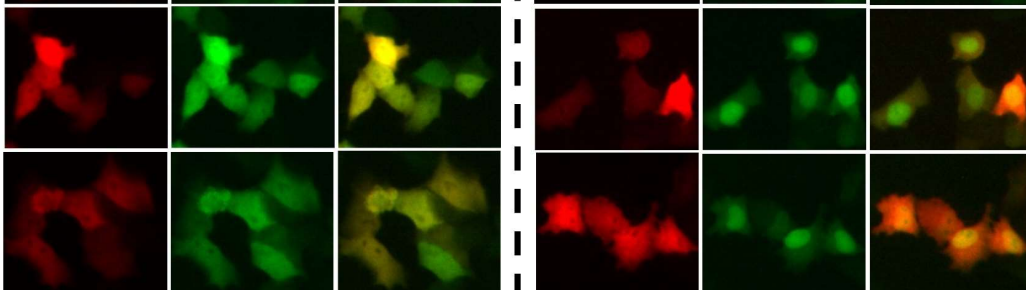

4WEN con
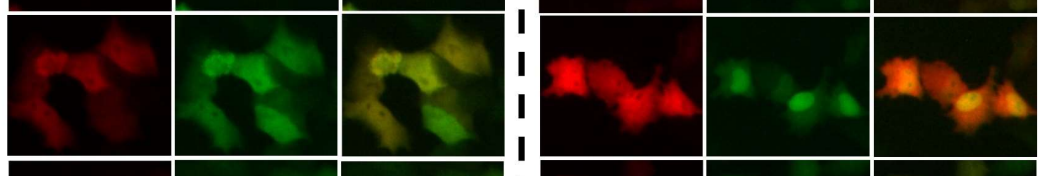

$3 \mathrm{~K} 74$ con
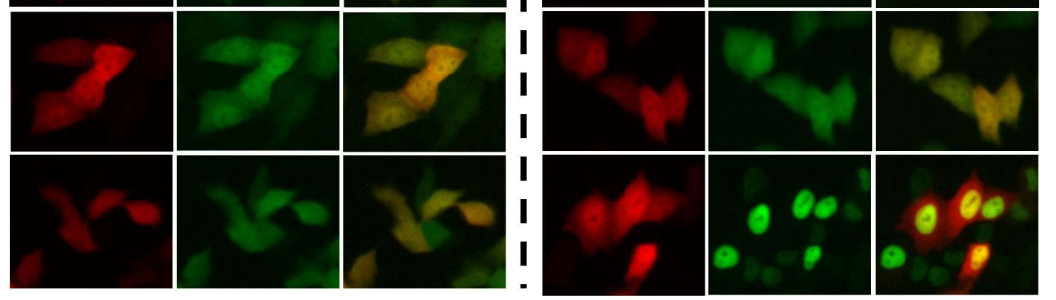

Anti-faeg
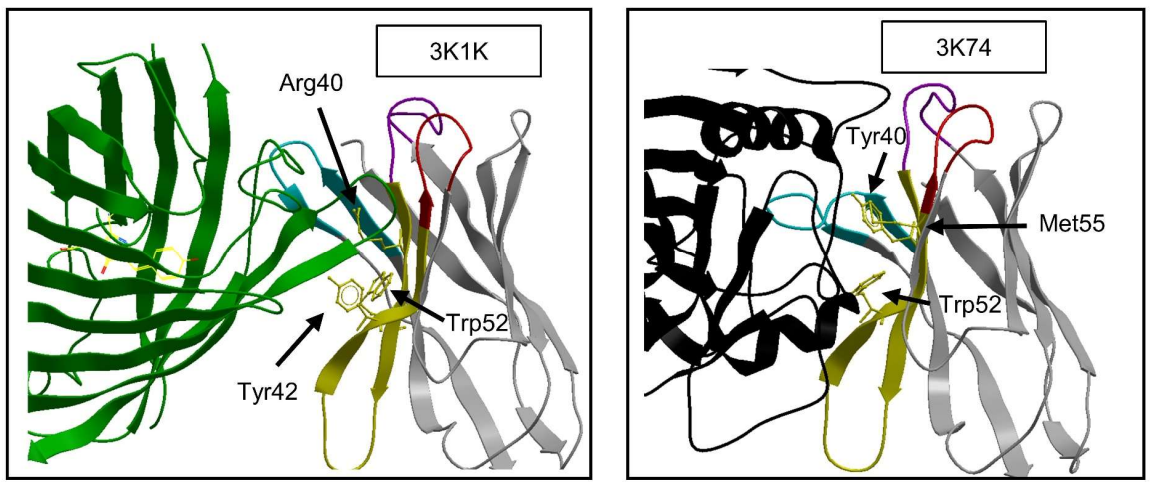

D

\begin{tabular}{l|c|c|c|c|c|c|c|}
\multicolumn{1}{c}{} & \multicolumn{1}{c}{ FR1 } & \multicolumn{1}{c}{ CDR1 } & FR2 & \multicolumn{1}{c}{ CDR2 } & FR3 & CDR3 & FR4 \\
\cline { 2 - 8 } 3G9A & 0 & 0 & 23 & 0 & 0 & 629 & 0 \\
\cline { 2 - 8 } 3K1K & 0 & 26 & 217 & 85 & 0 & 316 & 13 \\
\cline { 2 - 8 } 4WEM & 0 & 9 & 14 & 114 & 88 & 537 & 0 \\
\cline { 2 - 8 } 4WEN & 0 & 5 & 7 & 46 & 39 & 518 & 0 \\
\cline { 2 - 8 } 3K74 & 0 & 83 & 140 & 96 & 51 & 147 & 0 \\
\cline { 2 - 8 } 4113 & 0 & 81 & 0 & 123 & 0 & 409 & 0 \\
\hline
\end{tabular}

\section{Figure 5| Target binding of nanobodies with full consensus frameworks}

(A) All positional amino acids captured in the full consensus sequence. (B) Representative images of full consensus framework (con) nanobody-TagBFP expression in HeLa cells in the presence and absence of nuclearly localized-target. Red signal is from co-transfected CAG-dsRed plasmid. Transfected DNA amount was normalized by addition of empty vector plasmid to transfection mix for the "no target" condition. (C) Crystal structures of nanobodies that lose target binding when mutated to adhere to a full consensus framework are shown. Non-consensus framework residues directly contributing to target interface are depicted. (D) Target-interfacing surface area values in square angstroms (rounded to whole numbers) across distinct regions for nanobodies tested for target binding are shown. Values are taken from buried surface area interface values made available through PDBE-PISA. Scale bar is $25 \mu \mathrm{m}$. 


\section{Nanobody Intracellular Stability In Vivo}

As many research and therapeutic applications of nanobody technology will require expression in vivo, we wanted to test whether improved nanobody intracellular stability established in cell lines would apply to in vivo settings. Electroporation of the retina in newborn mice is a means to deliver nanobody expression vectors to multiple cell types. Plasmids encoding wild-type or mutant 3V0A-TagBFP were injected into the subretinal space of postnatal day 2 (P2) mice, along with a dsRed control plasmid, and electroporation was carried out. Tissue was harvested at P12, and was fixed, sectioned, and imaged. Representative images are displayed in Figure 6A. Red fluorescent signal from the dsRed control plasmid delineated areas of successful electroporation. Little to no TagBFP signal was observed in cells electroporated with wild-type 3VOA-TagBFP. However, strong TagBFP signal was observed in cells electroporated with mutant 3VOA-TagBFP, mirroring observations in cultured cells. These cells included highly specialized sensory neurons (rod photoreceptors), interneurons (bipolar cells), and glia (Mueller glia), suggesting that mutationally stabilized nanobodies are stable in multiple, distinct cell types.

For additional context validation, we tested mutated nanobodies for stability in bacteria. Several groups have illustrated powerful applications taking advantage of nanobodies expressed in bacteria ${ }^{20,21,22}$. Bacteria offer a therapeutic modality, as they can be used as deliverable factories for nanobody production. Moreover, stable expression of nanobodies in bacteria would facilitate intra-bacterial studies, and may contribute to improved production yields for nanobody reagents produced in bacteria. Five mutationally stabilized nanobodies and their parents were tested for expression in BL21 E. coli. Nanobodies were fused C-terminally to mCherry and expression was assessed by fluorescence microscopy. All 5 wild-type nanobodies exhibited hallmarks of instability in bacteria (Figure 6B). 4/5 wild-type nanobodies exhibited protein aggregation as illustrated by focal inclusion bodies, in addition to diminished cytoplasmic fluorescence. 3V0A did not appear to aggregate, but exhibited low fluorescent signal. All 5 mutationally stabilized nanobody variants exhibited a marked increase in fluorescence level. $1 Z \mathrm{~V} 5,4 \mathrm{WEN}$, and 1RJC mutants showed no signs of protein aggregation, in contrast to their wild-type parents. Mutant 3G9A exhibited the most intense fluorescent signal, but also exhibited fluorescent inclusion bodies, possibly due to higher nanobody concentration. 
bioRxiv preprint doi: https://doi.org/10.1101/2021.04.06.438746; this version posted April 8, 2021. The copyright holder for this preprint (which was not certified by peer review) is the author/funder, who has granted bioRxiv a license to display the preprint in perpetuity. It is made available under aCC-BY 4.0 International license.

524

526

527

530

531

532

533

534

535

536

537

538

539

540

541

542

543

544

545

546

547

548

549

550

551

A

dsRed
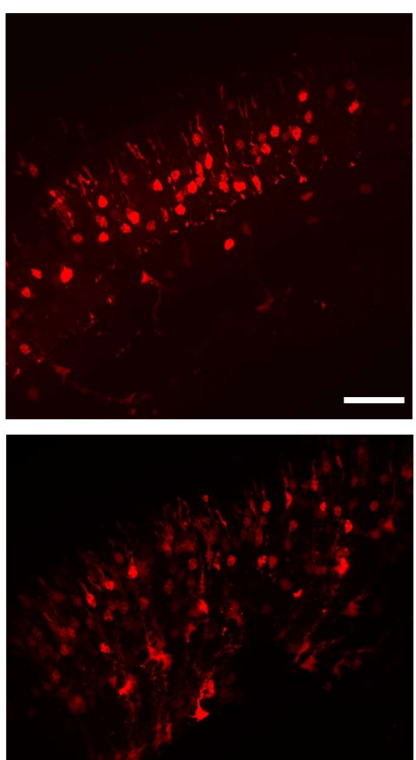

nb-tagBFP
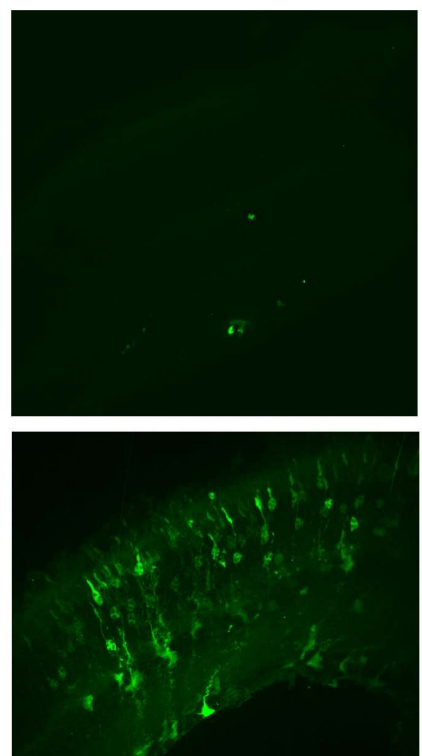

merge
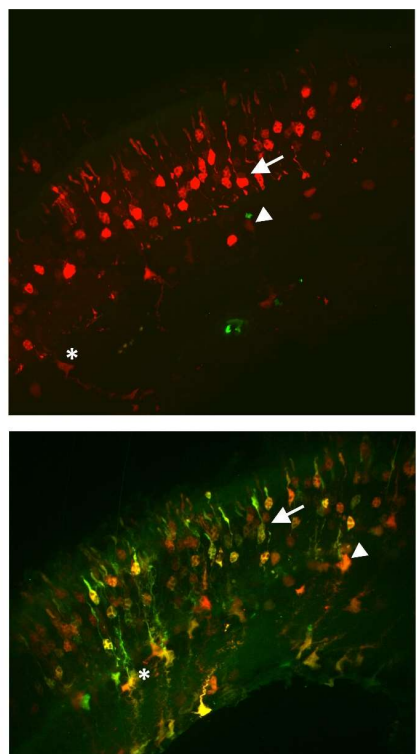

B
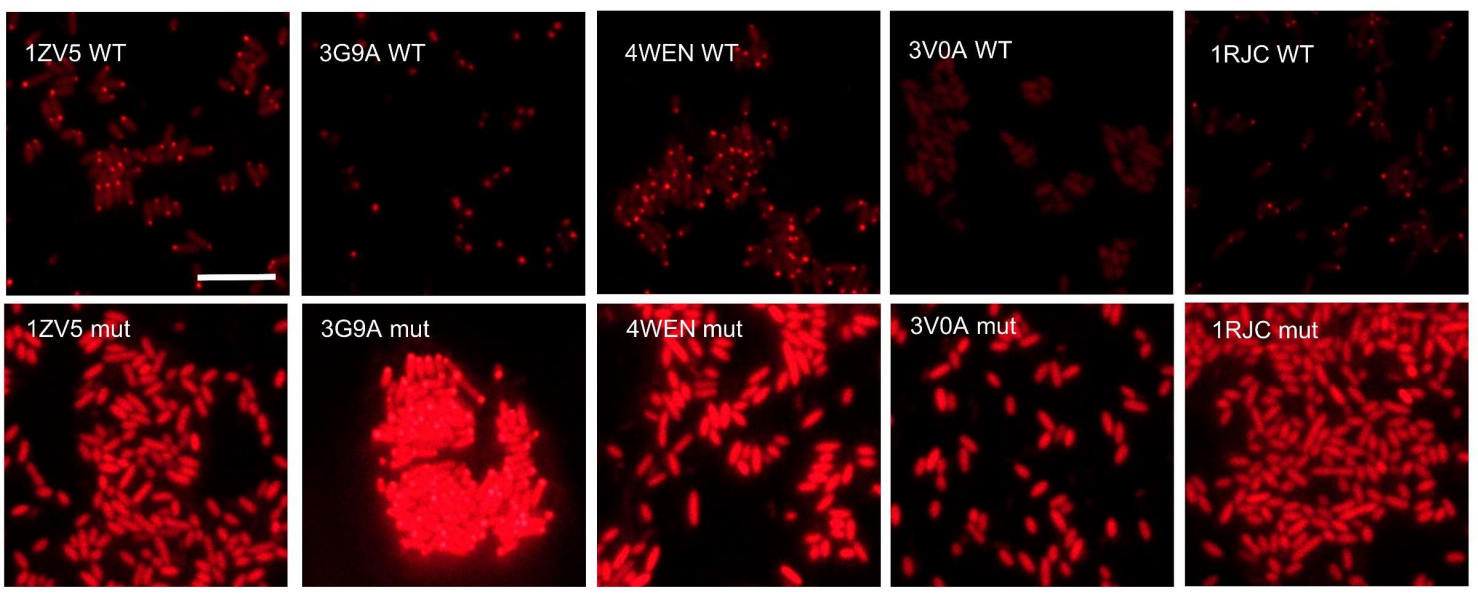

Figure 6| Nanobody Expression in the Murine Retina and E. Coli

(A) Representative Images of murine retina co-electroporated with CAG-dsRed and either wild-type or mutant CAG-3VOATagBFP plasmid. Retinas were electroporated on postnatal day 2 and harvested on postnatal day 12. Multiple cell types show expression following electroporation: photoreceptors (arrow), bipolar interneurons (triangle), and Mueller glia (asterix) are noted on merged images. (B) Representative images of BL21 E. coli transformed with either wild-type or mutant pRsetB-nanobody-mCherry plasmid. Images were taken following 4 hours of protein induction with $250 \mu \mathrm{M}$ IPTG. All scale bars are $10 \mu \mathrm{m}$. 


\section{Discussion}

Here, we describe a general and reliable method for modifying nanobody sequences to facilitate stable intracellular expression. A partial consensus framework was distilled from the most highly conserved positional residues across a large group of intracellularly stable nanobodies. This framework was applied broadly across unstable nanobodies to rescue intracellular stability in a great majority of cases. As new nanobodies are generated against intracellular targets, this stabilization approach should prove effective in achieving stable expression while maintaining target binding.

Consensus-based stabilization has been applied to antibody fragments in the past; Steipe and Wirtz applied consensus framework mutagenesis to $V_{L}$ and $V_{H}$ domains to enhance intracellular stability for conventional $F_{V}$ fragments ${ }^{23,24}$. This approach was successful as conventional antibodies are amenable for loop grafting onto full donor frameworks due to their near exclusive reliance on CDRs for binding. Our partial consensus approach takes into account variable binding modes observed across nanobody crystal structures. Nanobodies often rely on direct framework engagement for target binding. Omitting framework positions of high variability from mutagenesis is important for preserving target binding in some, perhaps many, cases.

While investigating intracellular target binding, we noted that some nanobodies were able to effectively bind their targets despite their observed instability when target is absent. Such nanobodies may be useful reagents without the need of stability engineering in certain cases. However, in cases of high nanobody expression and low target availability, an unstable, aggregation-prone fraction of unbound nanobody may prove problematic for studies concerning subcellular protein localization, or for reasons of cellular toxicity. Indeed, one potential therapeutic application for intracellular nanobodies is to block protein aggregation that leads to various neurodegenerative disease ${ }^{25}$. Several groups have investigated nanobodies as potential reagents for blocking aggregation of a range of cytotoxic, aggregation-prone factors including prion protein, amyloid beta, and alpha synuclein ${ }^{26,27,28}$. In these contexts, nanobody stability and solubility may prove critical to ensuring that no undue cellular stress is imparted by the treatment agent.

The potential for intracellular deployment of nanobody-based reagents is still far from being realized. Taking full advantage of the modularity and versatility inherent of these pareddown structures will require methods such as those proposed here to condition nanobodies for the intracellular environment. 


\section{Materials and Methods}

587

588

589

590

591

592

593

594

595

596

597

598

599

600

601

602

603

604

605

606

607

608

609

610

611

612

613

614

615

616

617

\section{Compilation of nanobody sequence and structure data}

Nanobody sequences were pulled directly from the PDB database (sequences for this study compiled in 2016). To generate an interface atlas profiling positions of interaction between each nanobody and its target (linked data provided), interface data were referenced from the Proteins, Interfaces, Structures, and Assemblies tool (PDBePISA), provided by the European Bioinformatics Institute (EMBL-EBI). Values for positional interactions were taken from Buried Surface Area values $\left(\AA^{2}\right)$ for each residue.

\section{$\underline{\text { Generation and cloning of nanobody sequences }}$}

Nanobody sequences were synthesized as double stranded DNA fragments (gBlocks) by IDT, and cloned directly into a CAG expression plasmid (Addgene plasmid 11150) (Matsuda and Cepko, 2004) in frame and linked with TagBFP via Gibson assembly (New England Biolabs, E2611). For bacterial expression constructs, nanobody sequences were PCR amplified with primers containing terminal regions of homology for cloning into a pRset plasmid (Addgene plasmid 3991)(Invitrogen, V35120) in frame and linked to mCherry. DH5a E. coli were transformed with assembled DNA and cultured in $4 \mathrm{~mL}$ cultures overnight in LB medium with 100 ug/mL carbenicillin. Plasmid DNA was purified using Qiagen miniprep kits.

Mutant nanobody sequences were designed by comparing intracellularly unstable nanobodies to a partial consensus framework sequence of stable nanobodies, and changing amino acids in each unstable nanobody to match the partial consensus sequence at each position. Nanobody framework regions were defined by The International Immunogenetics Information System (IMGT), a global reference for immunogenetics. The consensus sequence for stable nanobodies was generated by calculating the most frequent amino acid at each framework position across 33 intracellularly stable nanobodies. The partial consensus sequence represents the subset of consensus positions at which $80 \%$ or greater of the 33 stable nanobodies had the same amino acid (Figure 2B).

\section{$\underline{\text { Cell culture and transfection }}$}

All constructs used in mammalian cell transfection experiments were cloned into the pCAG plasmid. Plasmid DNAs encoding nanobody-TagBFP sequences were transfected into both 293T cells and HeLa cells, separately. Experiments were first conducted in 293T cells, validated across 3 replicates, and later verified in HeLa cells. 1 day prior to plasmid transfection, cells were plated in black, clear bottomed 96-well plates (CLS3603, Sigma-Aldrich) at roughly 10,000 
618

619

620

621

622

623

624

625

626

627

628

629

630

631

632

633

634

635

636

637

638

639

640

641

642

643

644

645

646

647

648

649

650

cells/well and incubated at $37^{\circ} \mathrm{C}$, and $5 \% \mathrm{CO}_{2}$. For transfection of cells in each well, $5 \mathrm{uL}$ of serum-free media was added to $200 \mathrm{ng}$ of plasmid DNA (100 ng CAG-nanobody-TagBFP plasmid and 100 ng CAG-dsRed plasmid (Addgene plasmid 11151) (Matsuda and Cepko, 2004)). For target-binding experiments, an additional $100 \mathrm{ng}$ of NLS-target expressing plasmid or $100 \mathrm{ng}$ of empty vector (control) was added. $1 \mathrm{uL}$ (or $1.5 \mathrm{uL}$ for target-binding experiments) of $1 \mathrm{mg} / \mathrm{mL}$ PEI (Polysciences, 24765-2) dissolved in water was added to DNA in media and vortexed for 10 seconds. Resulting transfection mix was left to sit at room temperature for 10 minutes before being added to cells.

\section{Live fluorescent imaging and stability scoring}

For initial stability scoring, 75 nanobodies were transfected into HEK293T cells (as described above) as TagBFP fusions driven by a CAG promoter. Roughly 24 hours after transfection, TagBFP signal was evaluated using a Leica DMI3000B microscope and a 20X objective lens. CAG-dsRed signal served as both a transfection control and orienting signal to assess cellular morphology. The 75 nanobodies were binned broadly into "stable" and "unstable" groupings based on the observed character of the TagBFP signal for each nanobody. Nanobodies with TagBFP signal that filled cells, mirroring dsRed signal, and that exhibited, at most, only minor and infrequent fluorescent puncta, were binned into the "stable" group. Nanobodies with sparse to absent TagBFP signal, and/or that exhibited major and frequent fluorescent puncta, were binned into the "unstable" group (Figure 1A). Groupings were validated across 3 separate rounds of transfection and evaluation in HEK293T cells, and further validated in HeLa cells. Grouping criteria were assigned to capture major differences in intracellular expression between groups, rather than subtle differences in expression pattern or character within groups.

For target-binding experiments, images were taken using the same Leica DMI3000B microscope and 20X objective lens roughly 24 hours after transfection. Excitation time was kept consistent between samples.

Imaging of parent and mutant nanobodies to evaluate mutational stabilization was carried out via automated confocal imaging. Using a PE Opera high-throughput confocal imaging system (https://www.flyrnai.org/supplement/BRO_OperaHighContentScreeningSystem.pdf) provided by the Drosophila RNAi Screening Center (https://fgr.hms.harvard.edu/), live, transfected HEK293T cells were imaged in 96-well plates using a water-immersion 40X objective. 10 fields for image acquisition, standardized across each well-plate well, were assigned prior to imaging. Through an automated protocol, 6 Z-stacks were taken in each field 
651 in both red and blue channels, spanning 12 microns. Following image acquisition, max-

652 projection images were generated, and nanobody-TagBFP signal was evaluated.

653 Retinal electroporation

654 Wild-type and mutant CAG-3V0A-TagBFP plasmids were each electroporated along with 655 control CAG-dsRed plasmid (1.5 ug/mL DNA) into the retina of P2 mouse pups. 3 pups received 656 wild-type 3VOA plasmid, and 3 pups received mutant 3VOA plasmid. Electroporation was 657 executed according to the methodology described in Matsuda and Cepko, 2004.

Retinal histology and imaging

Electroporated retinas were harvested at P12. Retinas were fixed in $4 \%$ formaldehyde for 30 minutes, transferred to PBS for 10 minutes, and transferred to $15 \%$ sucrose in PBS for 30 minutes, all at room temperature. Fixed retinas were then embedded in OCT, flash frozen, and

662 stored at $-80^{\circ} \mathrm{C} .12 \mu \mathrm{m}$ sections were made using a cryostat, placed on glass slides, and

663 mounted with Fluoromount-G mounting media (ThermoFisher, 00-4958-02). Images were taken

664 using a Nikon Ti2 inverted microscope (spinning disk confocal) with a 40X oil immersion

665 objective lens.

666

667

668

669

670

671

672

673

674

675

676

677

678

679

680

681

682

Bacterial expression and imaging

Nanobody sequences were cloned into the bacterial expression vector pRsetB in frame and fused to mCherry. Plasmid was transformed into BL21(DE3) E. coli (New England Biolabs, C2527), and individual colonies were picked into $5 \mathrm{~mL} \mathrm{LB}$ and cultured overnight at $37^{\circ} \mathrm{C}$ and shaken at 250 RPM. In the morning, $1 \mathrm{~mL}$ of each culture was added to $4 \mathrm{~mL}$ of M9 minimal media, and IPTG was added to a final concentration of $250 \mu \mathrm{M}$ to induce protein expression. Induction cultures were incubated for 4 hours at $37^{\circ} \mathrm{C}$ and 250 RPM. $40 \mathrm{uL}$ of induced culture was pipetted onto $3 \%$ M9-agar on a glass slide and covered with a glass coverslip. Bacteria were imaged with a Nikon Eclipse e1000 microscope using a 60X oil immersion objective lens. All images were taken with consistent excitation time. Experiments were validated across 3 replicates.

Image processing

Images were processed using ImageJ. Images from Figures 1 and 3 received the following processing and no other adjustments: 1) images were converted to 8-bit, 2) $Z$ stacks for each channel (red and blue) were merged to create maximum $Z$ projections, 3) maximum $Z$ projections for each channel were merged with one another, with red images inserted into the red channel, and blue images inserted into the green channel for greater contrast. Retinal 
683

684

685

686

687

688

689

690

691

692

693

694

695

696

697

698

699

700

701

702

703

704

705

706

707

708

709

710

711

712

713

714

715

716

images (Figure 6) received the same processing, but received additional contrast adjustment (all images received consistent adjustment to contrast). Other images, including bacterial images (images from Figures 4-6) without $Z$ stacks were similarly converted to 8-bit, were colorchannel merged, and received contrast equally administered contrast adjustment.

References:

1. Hamers-Casterman, C., Atarhouch, T., Muyldermans, S., Robinson, G., Hammers, C., Songa, E. B., Bendahman, N., \& Hammers, R. (1993). Naturally occurring antibodies devoid of light chains. Nature, 363(6428), 446-448. https://doi.org/10.1038/363446a0

2. Muyldermans, S. (2013). Nanobodies: natural single-domain antibodies. Annual Review of Biochemistry, 82(1), 775-797. https://doi.org/10.1146/annurev-biochem-063011092449

3. Steyaert, J., \& Kobilka, B. K. (2011). Nanobody stabilization of G protein-coupled receptor conformational states. Current Opinion in Structural Biology, 21(4), 567-572. https://doi.org/10.1016/j.sbi.2011.06.011

4. Staus, D. P., Wingler, L. M., Strachan, R. T., Rasmussen, S. G. F., Pardon, E., Ahn, S., Steyaert, J., Kobilka, B. K., \& Lefkowitz, R. J. (2014). Regulation of $\beta 2-A d r e n e r g i c$ Receptor Function by Conformationally Selective Single-Domain Intrabodies. Molecular Pharmacology, 85(3), 472-481. https://doi.org/10.1124/mol.113.089516

5. Audenhove, I. V., \& Gettemans, J. (2016). Nanobodies as Versatile Tools to Understand, Diagnose, Visualize and Treat Cancer. EBioMedicine, 8, 40-48. https://doi.org/10.1016/j.ebiom.2016.04.028

6. Jovčevska, I., \& Muyldermans, S. (2020). The Therapeutic Potential of Nanobodies. BioDrugs, 34(1), 11-26. https://doi.org/10.1007/s40259-019-00392-z

7. Traenkle, B., Emele, F., Anton, R., Poetz, O., Haeussler, R. S., Maier, J., Kaiser, P. D., Scholz, A. M., Nueske, S., Buchfellner, A., Romer, T., \& Rothbauer, U. (2015).

Monitoring Interactions and Dynamics of Endogenous Beta-catenin With Intracellular Nanobodies in Living Cells. Molecular \& Cellular Proteomics, 14(3), 707-723. https://doi.org/10.1074/mcp.m114.044016

8. Maier, J., Traenkle, B., \& Rothbauer, U. (2015). Real-time analysis of epithelialmesenchymal transition using fluorescent single-domain antibodies. Scientific Reports, 5(1), 13402. https://doi.org/10.1038/srep13402

9. Buchfellner, A., Yurlova, L., Nüske, S., Scholz, A. M., Bogner, J., Ruf, B., Zolghadr, K., Drexler, S. E., Drexler, G. A., Girst, S., Greubel, C., Reindl, J., Siebenwirth, C., Romer, 
717

718

719

720

721

722

723

724

725

726

727

728

729

730

731

732

733

734

735

736

737

738

739

740

741

742

743

744

745

746

747

748

749

750

751

T., Friedl, A. A., \& Rothbauer, U. (2016). A New Nanobody-Based Biosensor to Study Endogenous PARP1 In Vitro and in Live Human Cells. PloS One, 11(3), e0151041. https://doi.org/10.1371/journal.pone.015104

10. Tang, J. C., Drokhlyansky, E., Etemad, B., Rudolph, S., Guo, B., Wang, S., Ellis, E. G., Li, J. Z., \& Cepko, C. L. (2016). Detection and manipulation of live antigen-expressing cells using conditionally stable nanobodies. ELife, 5, e15312. https://doi.org/10.7554/elife.15312

11. Tang, J. C. Y., Szikra, T., Kozorovitskiy, Y., Teixiera, M., Sabatini, B. L., Roska, B., \& Cepko, C. L. (2013). A Nanobody-Based System Using Fluorescent Proteins as Scaffolds for Cell-Specific Gene Manipulation. Cell, 154(4), 928-939. https://doi.org/10.1016/j.cell.2013.07.021

12. Tang, J. C. Y., Rudolph, S., Dhande, O. S., Abraira, V. E., Choi, S., Lapan, S. W., Drew, I. R., Drokhlyansky, E., Huberman, A. D., Regehr, W. G., \& Cepko, C. L. (2015). Cell type-specific manipulation with GFP-dependent Cre recombinase. Nature Neuroscience, 18(9), 1334-1341. https://doi.org/10.1038/nn.4081

13. Kunz, P., Flock, T., Soler, N., Zaiss, M., Vincke, C., Sterckx, Y., Kastelic, D., Muyldermans, S., \& Hoheisel, J. D. (2017). Exploiting sequence and stability information for directing nanobody stability engineering. Biochimica et Biophysica Acta (BBA) General Subjects, 1861(9), 2196-2205. https://doi.org/10.1016/j.bbagen.2017.06.014

14. Kunz, P., Zinner, K., Mücke, N., Bartoschik, T., Muyldermans, S., \& Hoheisel, J. D. (2018). The structural basis of nanobody unfolding reversibility and thermoresistance. Scientific Reports, 8(1), 7934. https://doi.org/10.1038/s41598-01826338-z

15. Davies, J., \& Riechmann, L. (1994). 'Camelising' human antibody fragments: NMR studies on VH domains. FEBS Letters, 339(3), 285-290. https://doi.org/10.1016/00145793(94)80432-x

16. Davies, J., \& Riechmann, L. (1995). Antibody VH Domains as Small Recognition Units. Bio/Technology, 13(5), 475-479. https://doi.org/10.1038/nbt0595-475

17. Mitchell, L.S. and Colwell, L.J. (2018). Analysis of nanobody paratopes reveals greater diversity than classical antibodies. Protein Engineering, Design and Selection. 31, 7-8, 267-275. https://doi.org/10.1093/protein/gzy017

18. Saerens, D., Pellis, M., Loris, R., Pardon, E., Dumoulin, M., Matagne, A., Wyns, L., Muyldermans, S., \& Conrath, K. (2005). Identification of a Universal VHH Framework to Graft Non-canonical Antigen-binding Loops of Camel Single-domain Antibodies. Journal of Molecular Biology, 352(3), 597-607. https://doi.org/10.1016/j.jmb.2005.07.038 
752

753

754

755

756

757

758

759

760

761

762

763

764

765

766

767

768

769

770

771

772

773

774

775

776

777

778

779

780

781

782

783

784

785

19. Braun, M. B., Traenkle, B., Koch, P. A., Emele, F., Weiss, F., Poetz, O., Stehle, T., \& Rothbauer, U. (2016). Peptides in headlock - a novel high-affinity and versatile peptidebinding nanobody for proteomics and microscopy. Scientific Reports, 6(1), 19211. https://doi.org/10.1038/srep19211

20. Harmsen, M. M., Solt, C. B. van, Bemmel, A. M. van Z., Niewold, T. A., \& Zijderveld, F. G. van. (2006). Selection and optimization of proteolytically stable llama single-domain antibody fragments for oral immunotherapy. Applied Microbiology and Biotechnology, 72(3), 544-551. https://doi.org/10.1007/s00253-005-0300-7

21. Vandenbroucke, K., Haard, H. de, Beirnaert, E., Dreier, T., Lauwereys, M., Huyck, L., Huysse, J. V., Demetter, P., Steidler, L., Remaut, E., Cuvelier, C., \& Rottiers, P. (2010). Orally administered $\mathrm{L}$. lactis secreting an anti-TNF Nanobody demonstrate efficacy in chronic colitis. Mucosal Immunology, 3(1), 49-56. https://doi.org/10.1038/mi.2009.116

22. Chowdhury, S., Castro, S., Coker, C., Hinchliffe, T. E., Arpaia, N., \& Danino, T. (2019).

Programmable bacteria induce durable tumor regression and systemic antitumor immunity. Nature Medicine, 25(7), 1057-1063. https://doi.org/10.1038/s41591-019-0498Z

23. Ohage, E. C., Wirtz, P., Barnikow, J., \& Steipe, B. (1999). Intrabody construction and expression. II. A synthetic catalytic Fv fragment11Edited by R. Huber. Journal of Molecular Biology, 291(5), 1129-1134. https://doi.org/10.1006/jmbi.1999.3020 24. Wirtz, P., \& Steipe, B. (1999). Intrabody construction and expression III: Engineering hyperstable VH domains. Protein Science, 8(11), 2245-2250. https://doi.org/10.1110/ps.8.11.2245

25. Messer, A., \& Joshi, S. N. (2013). Intrabodies as Neuroprotective Therapeutics. Neurotherapeutics, 10(3), 447-458. https://doi.org/10.1007/s13311-0130193-6

26. Abskharon, R. N. N., Giachin, G., Wohlkonig, A., Soror, S. H., Pardon, E., Legname, G., \& Steyaert, J. (2014). Probing the N-Terminal $\beta$-Sheet Conversion in the Crystal Structure of the Human Prion Protein Bound to a Nanobody. Journal of the American Chemical Society, 136(3), 937-944. https://doi.org/10.1021/ja407527p

27. Kasturirangan, S., Boddapati, S., \& Sierks, M. R. (2010). Engineered Proteolytic Nanobodies Reduce $A \beta$ Burden and Ameliorate $A \beta$-Induced Cytotoxicity. Biochemistry, 49(21), 4501-4508. https://doi.org/10.1021/bi902030m 28. Chatterjee, D., Bhatt, M., Butler, D., Genst, E. D., Dobson, C. M., Messer, A., \& Kordower, J. H. (2018). Proteasome-targeted nanobodies alleviate pathology and 
786

787

788

789

790

791

792

793

794

795

796

797

798

799

800

801

802

803

804

805

806

807

808

809

810 functional decline in an a-synuclein-based Parkinson's disease model. Npj Parkinson's Disease, 4(1), 25. https://doi.org/10.1038/s41531-018-0062-4

29. Matsuda, T. and Cepko, C.L. (2004). Electroporation and RNA interference in the rodent retina in vivo and in vitro. Proceedings of the National Academy of Sciences. 101, 1, 1622. https://doi.org/10.1073/pnas.2235688100 
bioRxiv preprint doi: https://doi.org/10.1101/2021.04.06.438746; this version posted April 8, 2021. The copyright holder for this preprint (which was not certified by peer review) is the author/funder, who has granted bioRxiv a license to display the preprint in perpetuity. It is made available under aCC-BY 4.0 International license.

\section{Supplement:}

812

813 Supplemental Table 1: list of nanobodies and their associated antigens

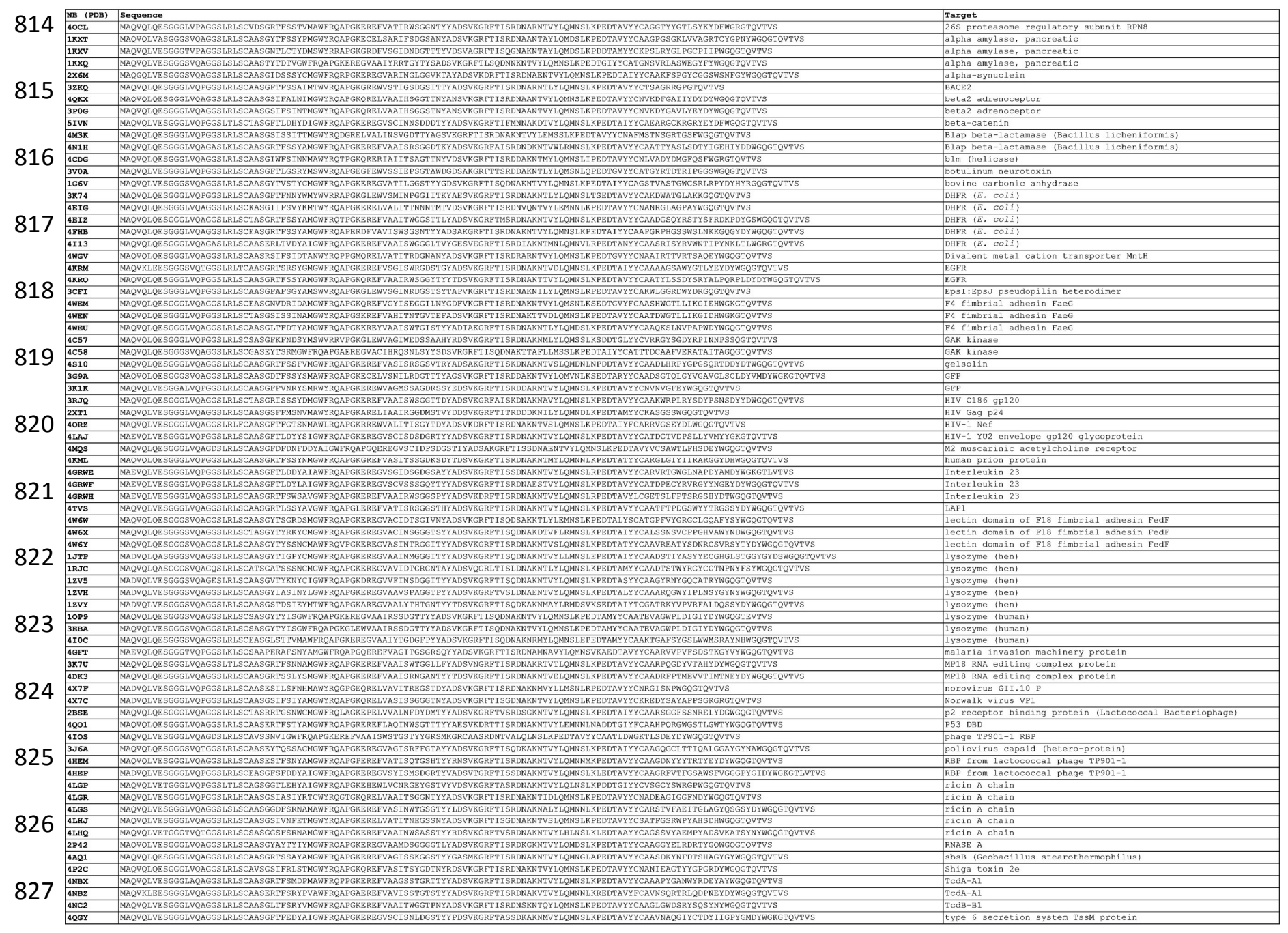

828

829

830

831

832

833

834 
bioRxiv preprint doi: https://doi.org/10.1101/2021.04.06.438746; this version posted April 8, 2021. The copyright holder for this preprint (which was not certified by peer review) is the author/funder, who has granted bioRxiv a license to display the preprint in perpetuity. It is made available under aCC-BY 4.0 International license.

3G9A WT

4WEM WT

3G9A mut

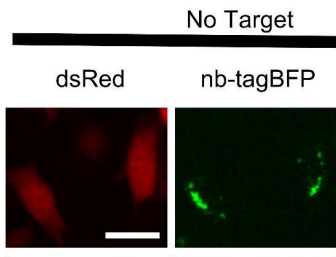

merge
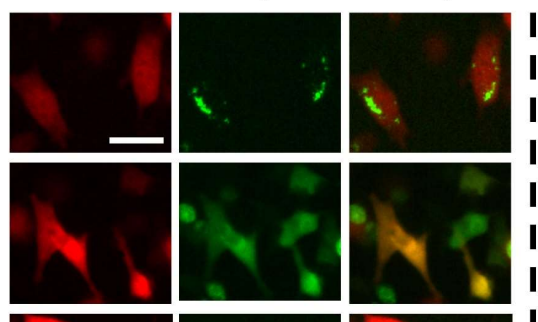

3K1K WT
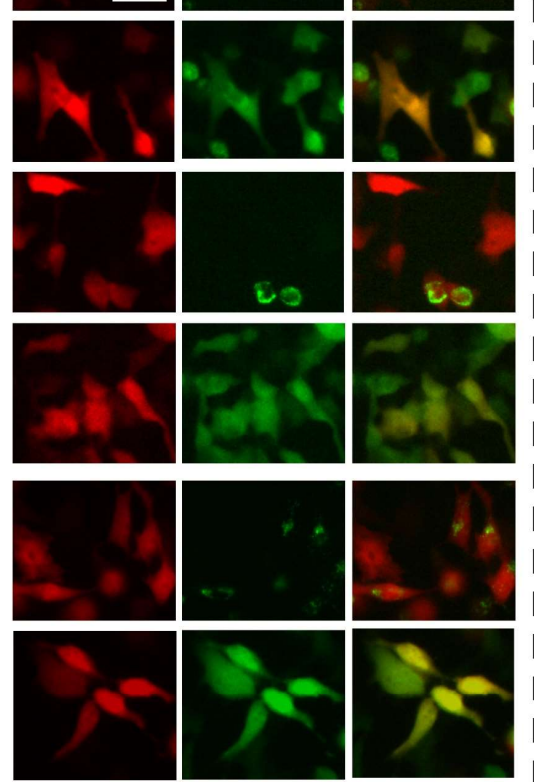

4WEN WT
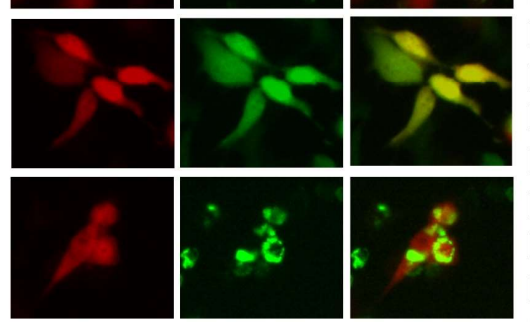

4WEN mut
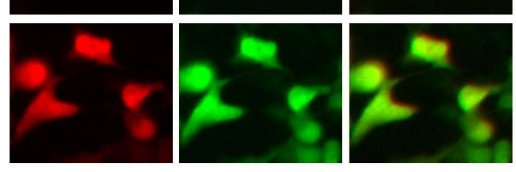

3K74 WT
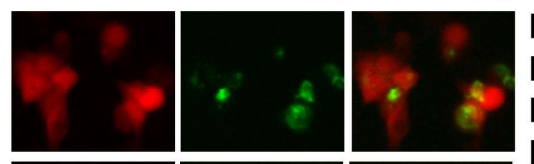

3K74 mut
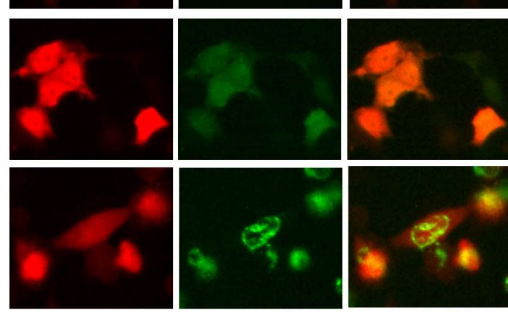

858

4113 mut
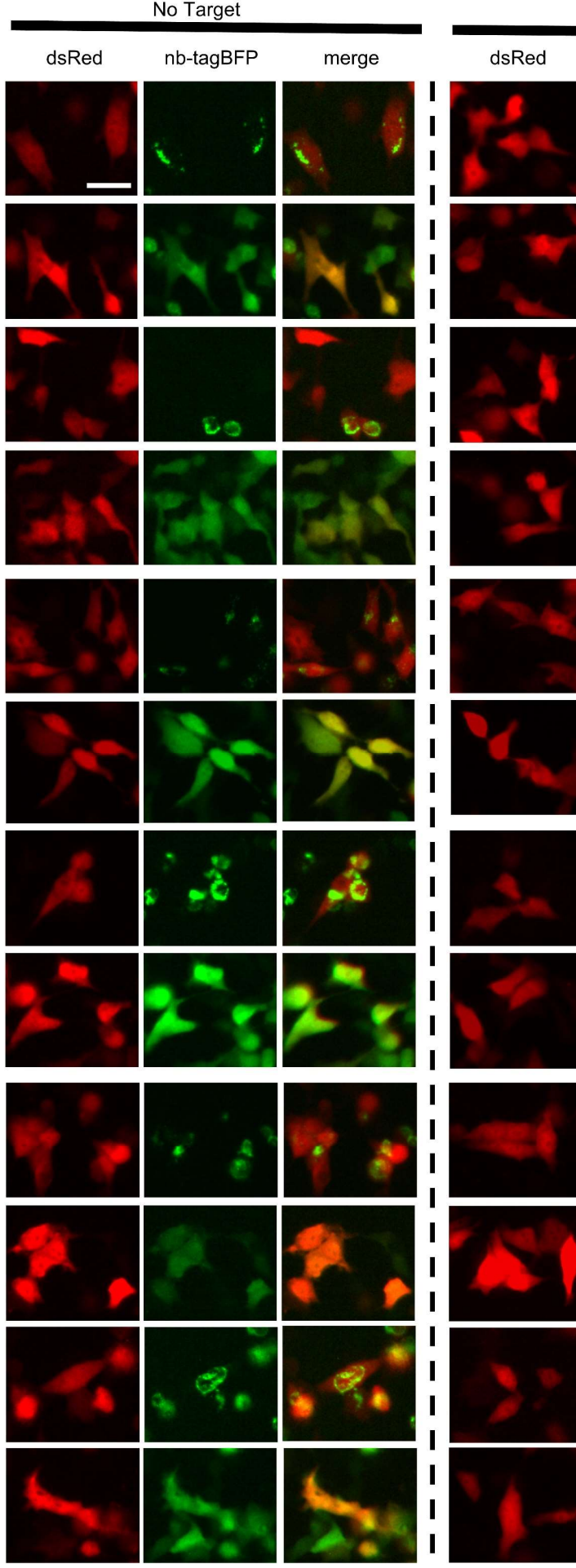

+ NLS-Target

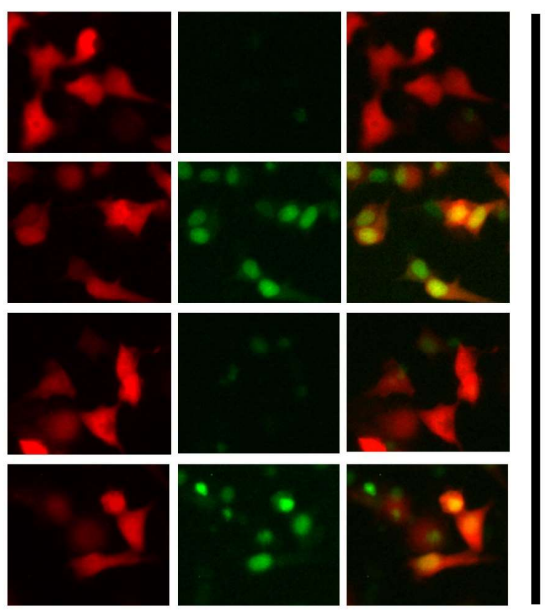

Anti-GFP

Anti-faeg
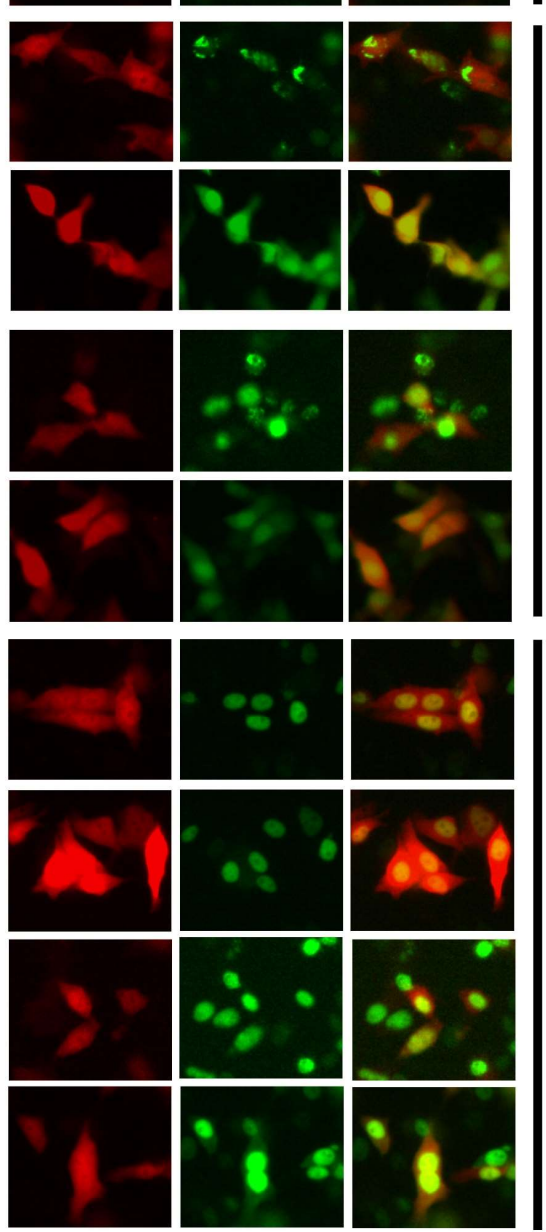

Anti-DHFR

(E. coli)

861

Representative images nanobody-TagBFP expression in HeLa cells in the presence and absence of nuclearly localizedtarget for all 6 nanobodies tested. Wild-type and mutant nanobodies are depicted. Red signal is from co-transfected CAGdsRed plasmid. Transfected DNA amount was normalized by addition of empty vector plasmid to transfection mix for the "no target" condition. Scale bar is $25 \mu \mathrm{m}$. 